\title{
Pulmonary and Systemic Toxicity in a Rat Model of Pulmonary Alveolar Proteinosis Induced by Indium-Tin Oxide Nanoparticles
}

\author{
Nan Liu', Yi Guan', Chunling Zhou², Yongheng Wang', Zhanfei Ma ${ }^{3}$, Sanqiao Yao ',4 \\ 'School of Public Health, North China University of Science and Technology, Tangshan, 0632 I0, Hebei, People's Republic of China; ${ }^{2}$ Baoding Center \\ for Disease Control and Prevention, Baoding, 071030, Hebei, People's Republic of China; ${ }^{3}$ Institute of Industrial Hygiene of Ordnance Industry, Xian, \\ 710065, Shanxi, People's Republic of China; ${ }^{4}$ XinXiang Medical University, Xinxiang, 453003, Henan, People's Republic of China
}

Correspondence: Sanqiao Yao, North China University of Science and Technology, Bohai Avenue 21, Tangshan, 063210, Hebei, People's Republic of China, Fax +86-3I5-8805583, Email sanqiaoyao@xxmu.edu.cn

Purpose: The main objective of this study was to clarify the biodistribution and in vivo toxicological effects of indium-tin oxide nanoparticles (Nano-ITO) in male rats.

Methods: Dose-response (three divided doses) and time-course studies (six exposure durations) were performed to examine Nano-ITO-induced pulmonary and systemic toxicity. At the end of the experiment, hematology and serum biochemical parameters were determined, and cytokines levels and oxidative stress were analyzed in the bronchoalveolar lavage fluid. In addition, indium biodistribution following Nano-ITO exposure was determined using inductively coupled plasma mass spectrometer to measure indium concentration in the lung, spleen, brain, liver, kidney, and testis. Rat lung tissues were also harvested for staining with hematoxylin and eosin, periodic acid Schiff stain, Masson's trichrome, and Sirius red.

Results: Relative lung weights were significantly increased in all Nano-ITO-exposed groups. All organs exhibited a statistically significant difference in indium levels. Rat exposure to Nano-ITO resulted in a dose-response increase in acute systemic inflammation and injury. BALF analysis revealed significantly elevated levels of lung oxidative stress, pulmonary injury, and inflammatory markers across most groups. Serum biochemistry results showed that Nano-ITO could affect the liver and renal functions of rats when exposed for 3 days. Compared with the control group, significant inflammatory responses or pathological changes were observed in the liver, kidney, and testis of rats at different sampling times and three doses examined. Histopathologically, foci of slight-to-severe pulmonary inflammatory response along with acute inflammatory, pulmonary fibrosis and alveolar proteinosis were detected, and the severity of these lesions worsened in a dose- and time-dependent manner.

Discussion: These findings provide novel evidence that enhanced progressive massive pulmonary fibrosis, diffuse interstitial fibrosis, and collagen accumulation play a role in the development of pulmonary alveolar proteinosis following Nano-ITO exposure.

Keywords: indium-tin oxide nanoparticles, pulmonary fibrosis, pulmonary alveolar proteinosis

\section{Introduction}

Indium-tin oxide (ITO), which is sintered at high temperatures using $90 \%$ indium oxide $\left(\operatorname{In}_{2} \mathrm{O}_{3}\right)$ and $10 \%$ tin oxide $\left(\mathrm{SnO}_{2}\right)$, is used as a transparent conductive coating for the manufacture of electronics such as liquid-crystal displays. In recent years, the potential health hazards of occupational exposure to potentially toxic particles generated during ITO manufacture have raised considerable concern, given the increasing demand for ITO production. The growth of the ITO industry has resulted in the emergence of indium lung disease, an occupational illness that has adversely affected the health of workers exposed to indium-containing compounds. Over the last decade, multiple case reports have documented lung injury and inflammation, pulmonary fibrosis, emphysema, pulmonary alveolar proteinosis (PAP), and even lung cancer in workers exposure to indium and its compounds. ${ }^{1-9}$ Consequently, it is important to understand the potentially toxic effects of indium and related compounds. 
Although the toxicity of indium and its compounds (especially ITO) has been examined in detail following individual exposures, ${ }^{10-15}$ to the best of our knowledge, limited data are available on the pulmonary and systemic toxicity of ITO nanoparticles (Nano-ITO). Thus, there is an urgent need to determine whether Nano-ITO could induce pulmonary and systemic toxicity in vivo. To address this issue, we investigated and compared the pulmonary effects of Nano-ITO in rat exposed to three doses and six distinct time points. The findings of this study could provide a broader picture reflecting the overall effects, including the body's adaptations to injury, establishing basic toxicological data for health risk assessments following human exposure to Nano-ITO. Simultaneously, we aimed to evaluate the in vivo toxicological effects of repeated intratracheal Nano-ITO instillation through the comparative analysis of lung, spleen, brain, liver, kidney and testis toxicity. Our study combines molecular, biochemical, and histological approaches and, thus, contributes to a more complete and comprehensive understanding of Nano-ITO toxicological profile.

\section{Materials and Methods}

\section{Animals}

Four-week-old male specific-pathogen-free Sprague-Dawley (SD) rats, weighing 160-190 g, were purchased from the Medical Laboratory Animal Center of North China University of Science and Technology. The rats were reared in a ventilated, temperature-controlled, and standardized sterile animal room (temperature of $20 \pm 2{ }^{\circ} \mathrm{C}$, relative humidity of $60 \pm 10 \%$ ), with a $12 \mathrm{~h}$ day/light circle at the School of Public Health. Food (sterile stock diet) and water (via an automated watering system) were provided ad libitum. The rats were adapted to the animal facility for 1-2 weeks prior to experimentation and monitored daily for general health. All procedures used in this study were conducted in accordance with the Guide for the Care and Use of Laboratory Animals Center of North China University of Science and Technology and approved by the Experimental Animal Ethics Committee of North China University of Science and Technology (Permit No. 2017011).

\section{ITO Nanoparticle Preparation}

Nano-ITO, with a mean diameter of $50 \mathrm{~nm}$, were provided by Aladdin, China and contained $74.4 \%$ (wt $\%$ ) indium and $7.8 \%$ tin, with the remainder composed of oxygen. Nano-ITO were suspended in sterile physiological saline immediately prior to each intratracheal instillation. The microstructure, surface morphology and relative size of Nano-ITO were determined using scanning electron microscopy (SEM). SEM measurements were performed on a SIGMA HD S4800 (USA), operated at an accelerating voltage of $5 \mathrm{kV}$. The size and morphology of Nano-ITO in lung tissue were characterized using transmission electron microscopy (TEM; H-7650, HITACHI, Japan) with an acceleration voltage of $80 \mathrm{kV}$. Zeta potential and hydrodynamic diameters were characterized by zeta potential and particle size analyzer (Zetasizer Nano ZS, Malvern, UK).

\section{Experimental Design}

We selected an intratracheal instillation model rather than an inhalation study, as the former is an easy and reliable method to identify nanoparticle toxicity. Briefly, rats were deeply anesthetized with isoflurane. Then a plastic catheter was used for tracheal intubation, and the Nano-ITO suspension was instilled at a volume of $1 \mathrm{~mL}$. The study design is presented in Figure 1. Male SD rats were grouped randomly.

\section{Dose-Response Study}

Rats were intratracheally instilled $0,1.2,3$, and $6 \mathrm{mg} / \mathrm{kg}$ body weight of Nano-ITO in $1 \mathrm{~mL}$ of physiological saline using a syringe, twice a week, and sacrificed at week 12 post-exposure.

\section{Time-Course Study}

Rats were divided into six Nano-ITO-exposed groups and six control groups, corresponding to the respective Nano-ITOexposed groups. Each rat was intratracheally instilled with $6 \mathrm{mg} / \mathrm{kg}$ Nano-ITO, twice weekly. The dispersed Nano-ITO was administered within 1 min of ultrasonication. Control group animals were administered physiological saline 


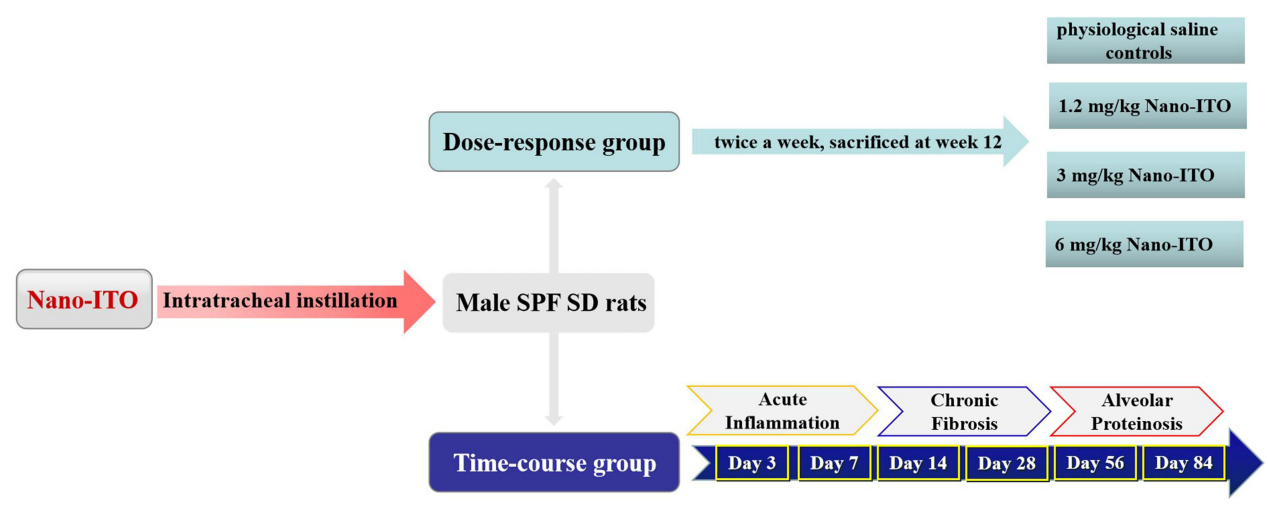

Figure I Schematic diagram of male SD rat experimental design. Ten rats were used in each group and two independent experiments were performed accordingly.

intratracheally at the same frequency and timing as Nano-ITO-exposed groups. To examine the short- and medium-term effects, rats were sacrificed on days 3, 7, 14, 28, 56 and 84 after intratracheal instillation.

\section{Experimental Processing}

Survival, food intake, fur, behavior, mental status, urine, and feces were recorded daily for each experimental animal. The body weights were recorded every week. At predetermined time points (3, 7, 14, 28, 56 and 84 days), rats from each group were anesthetized using isoflurane. Whole blood was collected from the abdominal aorta and transferred to two collection tubes: a vacutainer containing EDTA (used for whole blood hematological analysis) and a vacutainer containing clot activator and a polymer gel (used for serum chemistry analysis). After clamping the left lung lobe and tying off the right cardiac lobe, bronchoalveolar lavage fluid (BALF) was collected from the remaining right lobes (BALF collection further described below). After the lavage, the tied-off right lung lobe and lavaged right lobes were collected and stored at $-80{ }^{\circ} \mathrm{C}$. Macroscopic examinations of the body surface, subcutis, and all internal organs in the head, abdominal, and thoracic cavities were performed. Subsequently, the lung, spleen, brain, liver, kidney and testis were harvested and weighed using an electronic balance. A portion of the obtained tissues was fixed in tissue fixative ( $10 \%$ neutral buffered formalin) for subsequent evaluation of histopathological changes. Other fresh tissue samples were stored at $-80{ }^{\circ} \mathrm{C}$ for measuring indium levels in organs. Weights were recorded both as absolute organ weight and as relative organ weight. The relative organ weight was calculated based on the ratio of absolute organ weight to fasted body weight.

\section{Blood Analysis}

\section{Hematological Parameters}

Hematology analysis included the following parameters: white blood cell (WBC), red blood cell (RBC), hemoglobin (HGB), platelet count (PLT), hematocrit (HCT), mean corpuscular volume (MCV), mean corpuscular hemoglobin $(\mathrm{MCH})$, mean corpuscular hemoglobin concentration (MCHC), RBC volume distributing width standard deviation (RDW-SD), RBC volume distributing width coefficient of variation (RDW-CV), platelet distribution width (PDW), mean platelet volume (MPV), platelet crit (PCL), percentage of large platelets (P-LCR), neutrophil (Neu) percentage, lymphocyte (Lym) percentage, determined using a ProCyte Dx Hematology Analyzer.

\section{Serum Chemistry Profile}

Serum biochemistry analysis was evaluated using a Catalyst One Chemistry Analyzer to determine the following parameters: total protein (TP), albumin (ALB), globulin (GLB), albumin and globulin ratio (A/G), alanine aminotransferase (ALT), aspartate transaminase (AST), cholinesterase (CHE), total bilirubin (TBIL), direct bilirubin (DBIL), and indirect bilirubin (IBIL). Blood urea nitrogen (BUN), uric acid (UA), creatinine (Cr), malondialdehyde (MDA), superoxide dismutase (SOD), hydroxyproline (HYP), Catalase (CAT), and glutathione peroxidase (GSH-px), were measured using kits from the Nanjing Jiancheng Bioengineering Institute (Nanjing, China). 


\section{Determination of Organs Indium Concentrations}

The lung, spleen, brain, liver, kidney and testis of each rat were digested in the microwave digestion instrument by

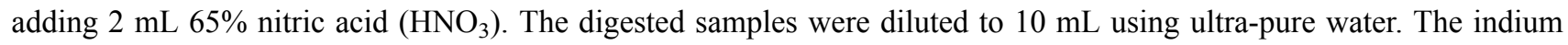
concentration in tissues was determined using an inductively coupled plasma mass spectrometer (ICP-MS 7500a, Agilent, USA). Rhodium was used as an internal standard for the indium measurement. The quantitative detection limit of indium was $0.045 \mu \mathrm{g} / \mathrm{g}$ for lung tissue. For all measurements, nitric acid blank and indium standards were prepared and concurrently tested with test samples. Tissues from the control group at different time points were dissolved similarly. The tissue concentration of indium was calculated using the following equations: [indium] treated tissue $(\mu \mathrm{g} / \mathrm{g}$ wet tissue $)=[$ indium $]$ tissue suspension/wet weight of tissue.

\section{Bronchoalveolar Lavage (BAL) Analysis BAL Fluid (BALF) Collection}

After exsanguination, the trachea was cannulated, the chest cavity opened, and BALF was collected from the right lung (except the cardiac lobe) via the tracheal cannula. The first BAL fraction was obtained by gently filling the right lung with $6 \mathrm{~mL}$ cold phosphate-buffered saline (PBS), massaging for 30s, withdrawing, and repeating the process for another 30s. The second BAL fraction consisted of subsequent aliquots of $5 \mathrm{~mL}$ PBS (instilled and withdrawn) until a $15 \mathrm{~mL}$ volume was obtained. Both BAL fractions were mixed and centrifuged $\left(800 \times \mathrm{g}\right.$ for $10 \mathrm{~min}$ at $\left.4{ }^{\circ} \mathrm{C}\right)$. The supernatant was stored at $-80{ }^{\circ} \mathrm{C}$ for subsequent analysis of parameters indicative of inflammation, injury, oxidant production, and immune function.

\section{SOD, Total Antioxidant Capacity (T-AOC), MDA, Total Protein and Lactate Dehydrogenase (LDH) Activity} To evaluate cytotoxicity as a measure of lung injury, The levels of LDH activity, SOD, T-AOC, MDA, and TP were measured in the BALF of all treatment groups at each time point after exposure. LDH activity in the BALF was measured using an LDH Cytotoxicity Detection Kit. The TP concentration in the BALF was determined by the Bradford method using Bio-Rad Protein Assay. TP was measured to assess the breakdown in the integrity of the alveolar-capillary barrier.

\section{BALF Cytokines Levels}

The levels of pro- and anti-inflammatory cytokines were measured from the undiluted first BAL fraction, including interleukin (IL)-1 $\beta$, IL-6, IL-10, and tumor necrosis factor (TNF)- $\alpha$, using enzyme-linked immunosorbent assay (ELISA) kits, according to the instructions provided.

\section{Histopathological Evaluation}

Five rats from each control group and five from each exposure group were used for gross and histopathological assessment. All rats underwent complete necropsy, with examinations of the external body surfaces and all orifices, as well as the cranial, thoracic, and abdominal cavities and their contents. The following organs were trimmed and weighed: lung, spleen, brain, liver, kidney and testis. Selected tissues sections were placed into embedding cassettes and fixed by immersion in $10 \%$ neutral-buffered formalin. The formalin-fixed tissues were routinely processed and embedded in paraffin for histopathological examination. Tissue sections (approximately 5 - $\mu \mathrm{m}$ thick) were cut and stained with hematoxylin and eosin (H\&E) to visualize inflammatory infiltrates. Furthermore, the embedded lung tissues were cut into 3- $\mu \mathrm{m}$ thick sections and stained with periodic acid Schiff (PAS) stain to visualize alveolar proteinosis, Masson's trichrome for lung fibrosis, or Sirius red staining to visualize collagen deposition in the lung. Tissue sections were evaluated by a board-certified veterinary pathologist using light microscopy.

\section{Statistical Analyses}

Differences among groups were evaluated by one-way analysis of variance (ANOVA) followed by Bonferroni $t$-test, Dunnett's $t$-test, or Dunn's method. If data were not normally distributed, Kruskal-Wallis ANOVA on Ranks was used. Data on body weight were analyzed by two-way repeated measure ANOVA. Fisher's least significant difference 
procedure was used for organ weight analysis after one-way ANOVA. Hematological and blood biochemical parameters were analyzed using Dunnett's test. Data are expressed as mean \pm standard error (SE) unless otherwise noted. For all statistical comparisons, a p-value $<0.05$ was used to determine significant differences. Statistical analyses were performed using IBM SPSS 21.0 (IBM Corp., Armonk, NY, USA).

\section{Results}

\section{Characterization of ITO Nanoparticles}

The size and surface morphology of Nano-ITO were examined using SEM. The SEM images are presented in Figure 2A and show that the Nano-ITO was nearly spherical or ellipsoidal in shape, uniformly sized in the region of $50 \mathrm{~nm}$. TEM revealed Nano-ITO aggregates, black dots, in the lung of rats exposed to Nano-ITO for 12 weeks (Figure 2B). The primary size of the Nano-ITO was $55.3 \pm 1.1 \mathrm{~nm}$, while the hydrodynamic diameter and zeta potential of Nano-ITO were $785.12 \pm 1.62 \mathrm{~nm}$, and $(-32.11 \pm 0.62) \mathrm{mV}$, respectively, indicating that the nanoparticles were agglomerated and can be easily dispersed by simple sonication. Aggregation is a well-documented ability of nanoparticle, and human exposure includes contact with aggregated nanoparticles to a certain extent; hence, it is relevant to assess aggregated nanoparticles in toxicological research.

\section{Body Weight and Organ Weight/BW Coefficients}

No rats died during the intratracheal instillation and observational period. No systemic signs such as respiratory distress, dermatological abnormality, or behavioral or neurological disorder were apparent during the observation period in any group examined. Following intrathecal instillation of Nano-ITO, the behavior, mental status, fur, food intake, urine, and feces of rats were observed weekly. No unusual changes were detected. The body weight of rats was continuously recorded for 12 weeks, and the data are shown in Figure 3A. The body weight of Nano-ITO-exposed groups and the control group showed similar increasing trends throughout the study. No statistically significant differences in body weight were observed between Nano-ITO groups and controls at any time point of necropsy. These results suggested that Nano-ITO did not interfere with the growth of rats. Following sacrifice at various intratracheal instillation times, primary organs were harvested and weighed carefully. The organ index of main organs was calculated as organ weight (mg)/body weight (g). The organ index of the lung, spleen, brain, liver, kidney and testis of rats at week 12 are shown in Figure 3B, revealing a statistically significant difference in lung index values between three doses of Nano-ITO-exposed groups and the control group. In addition, the Nano-ITO $6 \mathrm{mg} / \mathrm{kg}$ group presented a significantly higher lung index than the NanoITO $1.2 \mathrm{mg} / \mathrm{kg}$ and Nano-ITO $3 \mathrm{mg} / \mathrm{kg}$ groups. In each Nano-ITO group, the increased in relative lung index surged in a dose-dependent manner until the end of the observation period. Compared with the control group, the organ index of
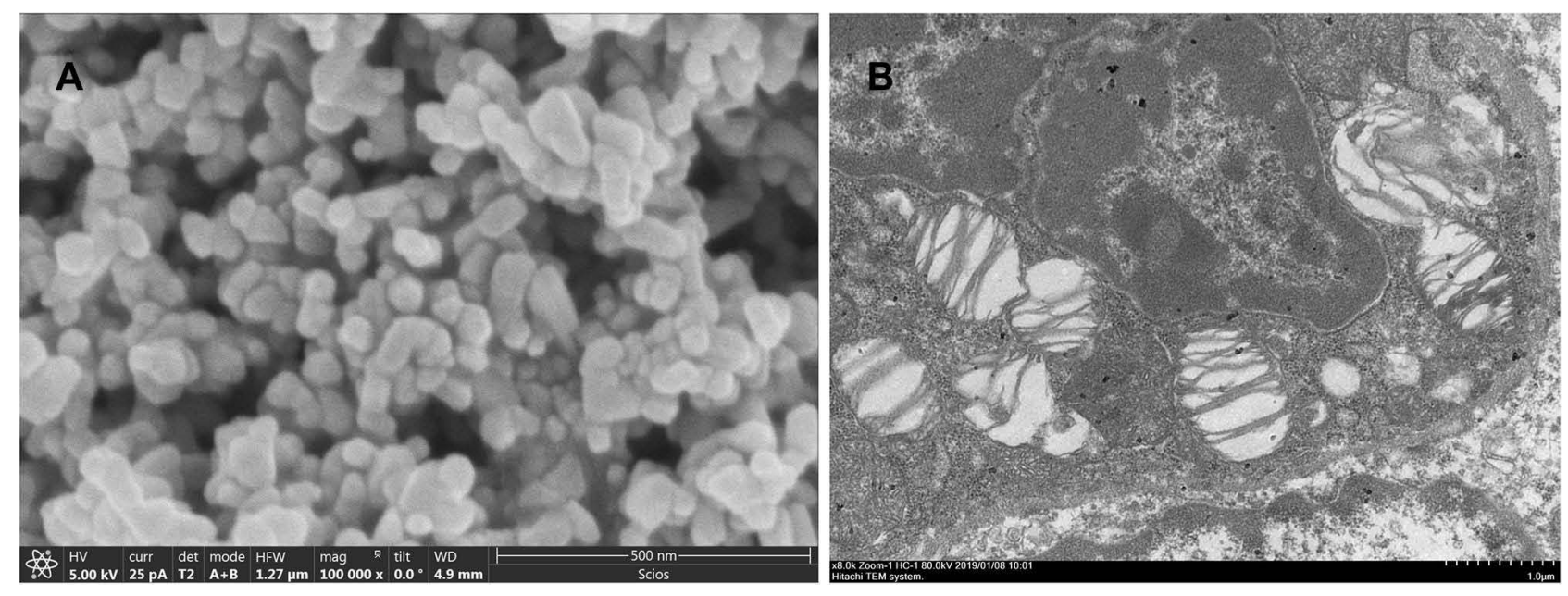

Figure 2 The characterization of the Nano-ITO. (A) The SEM images of the Nano-ITO, and the particles were in nearly spherical structure. Scale bar = 500 nm. (B) NanoITO bioaccumulate in lung tissues by TEM analysis. Scale bar $=1 \mu \mathrm{m}$. 


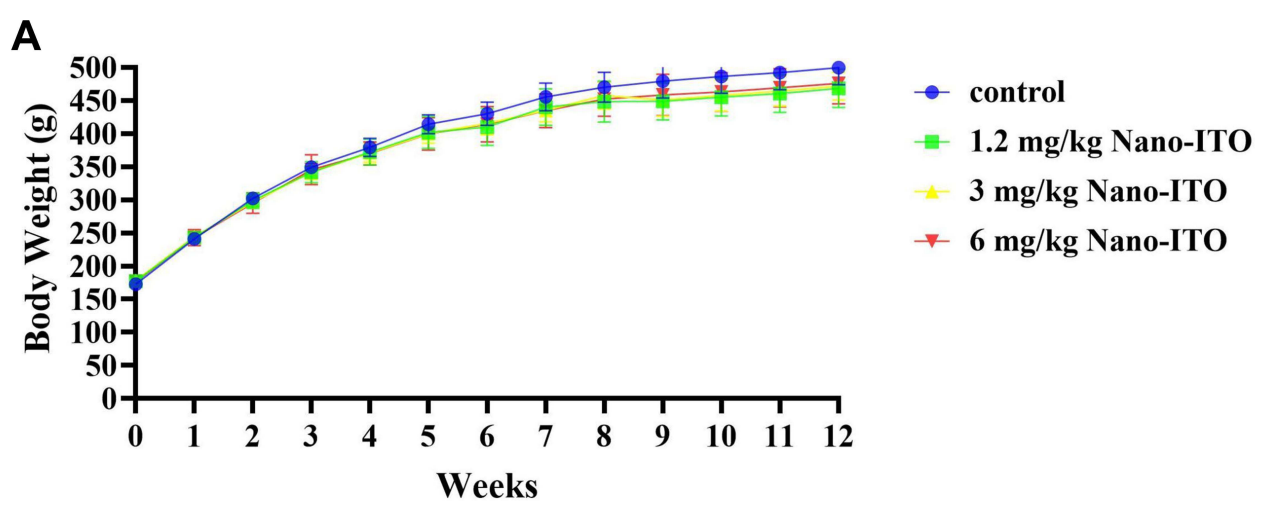

B

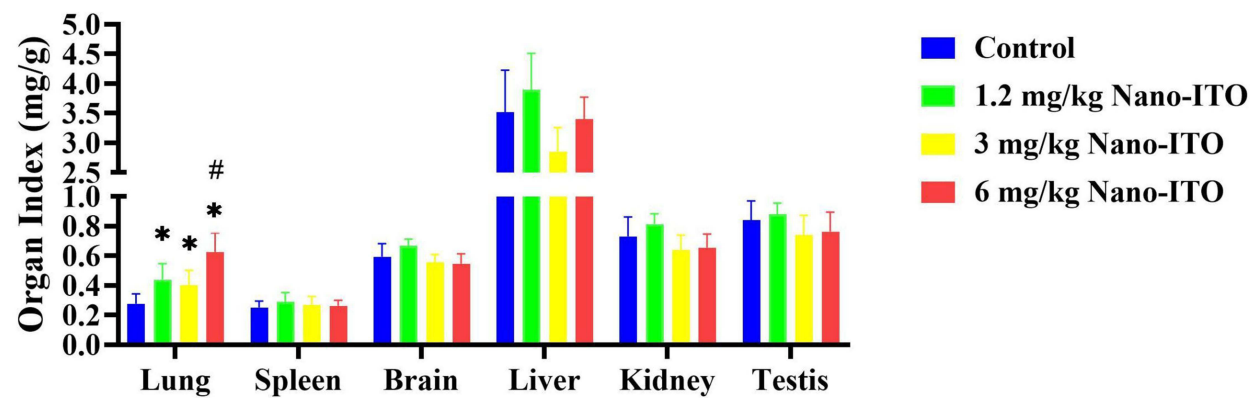

Figure 3 Body weight and organ index in rat. (A) The body weight curve of rat continuously monitored for 12 weeks. (B) The main organ index of rat on Week 12 after exposure to Nano-ITO. ${ }^{*} P<0.05$ vs control; ${ }^{*} P<0.05$ vs Nano-ITO $1.2 \mathrm{mg} / \mathrm{kg}$ and $3 \mathrm{mg} / \mathrm{kg}$ group.

the liver in the $3 \mathrm{mg} / \mathrm{kg}$ Nano-ITO group decreased significantly, whereas the organ index of the lung significantly increased.

\section{Dosimetry of Nano-ITO in Selected Tissues}

To quantitate the Nano-ITO accumulation in the lung, spleen, brain, liver, kidney and testis, the indium element concentration was measured in tissues at different exposure times and doses using ICP-MS. As shown in Figure 4, NanoITO showed a gradual increase in indium levels in tested organs and peaked in the high-dose group and at 84 days. Indium accumulation was the highest in the lung, followed by the spleen, liver, and kidney, and this result was consistent with the findings of Tanaka et al. ${ }^{16}$ In the study assessing different dose-exposed rats, indium concentrations of all organs measured by ICP-MS were significantly higher in rats necropsied immediately after exposure than in controls (Figure 4A). The concentration of indium element in the brain was approximately one-tenth of that in the liver and kidney. Relative to the control group, indium levels in the brain and testis were lower in Nano-ITO-exposed rats but gradually increased, exhibiting a sharp increase in the indium concentration and a tenfold higher indium content. In contrast with brain levels, the testis showed a 100-fold higher indium content. Indium levels increased in major organs of all exposure groups in a dose-dependent manner. At the end of the 84-day exposure period, indium accumulated as follows: lung $>$ spleen $>$ liver $>$ kidney $>$ testis $>$ brain.

On assessing rats subjected to different exposure times, indium concentrations in the liver and testis were elevated in the 3 days post-exposure group when compared with control rats, but this increase was not statistically significant (Figure 4B). Therefore, a statistically significant difference was observed in the indium levels of all organs, except for the liver and testis in the day 3 Nano-ITO group, when compared with those in the control group. The above results revealed that the order of Nano-ITO distribution in these tissues was lung, spleen, liver, kidney, testis, and brain, following intratracheal instillation. Furthermore, the lung of rats exposed to $6 \mathrm{mg} / \mathrm{kg}$ Nano-ITO showed a time-dependent increase in indium concentration. Although the results demonstrated that indium can accumulate in the body for a prolonged period and can be transferred from the lung to other organs and tissues, the clearance of indium from the body was 
A

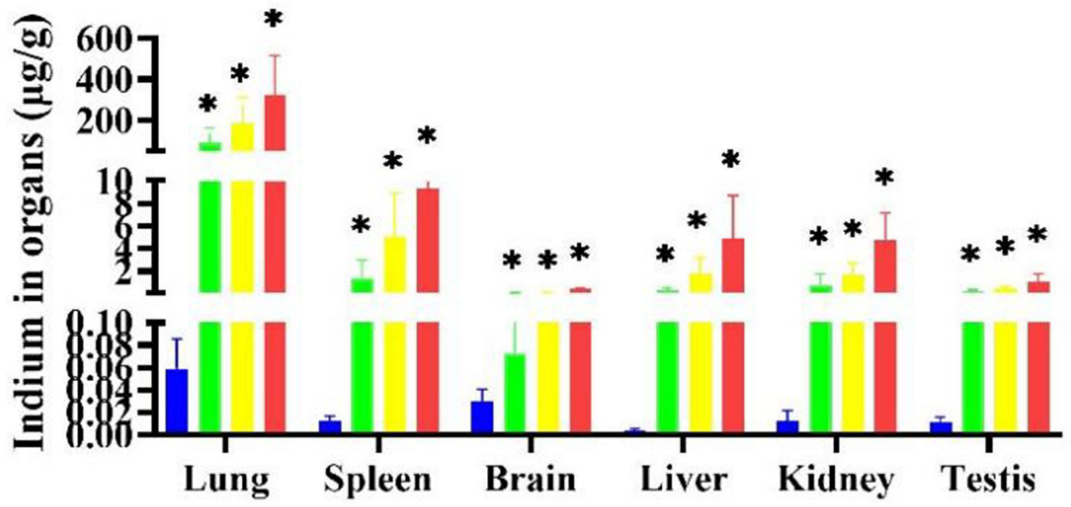

Control

\section{$1.2 \mathrm{mg} / \mathrm{kg}$ Nano-ITO}

$3 \mathrm{mg} / \mathrm{kg}$ Nano-ITO

$6 \mathrm{mg} / \mathrm{kg}$ Nano-ITO

\section{B}

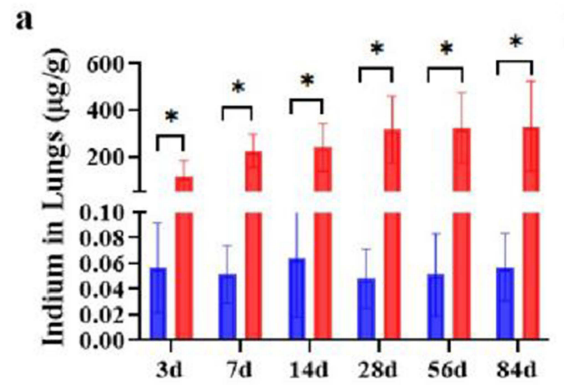

Control

$6 \mathrm{mg} / \mathrm{kg}$ Nano-ITO
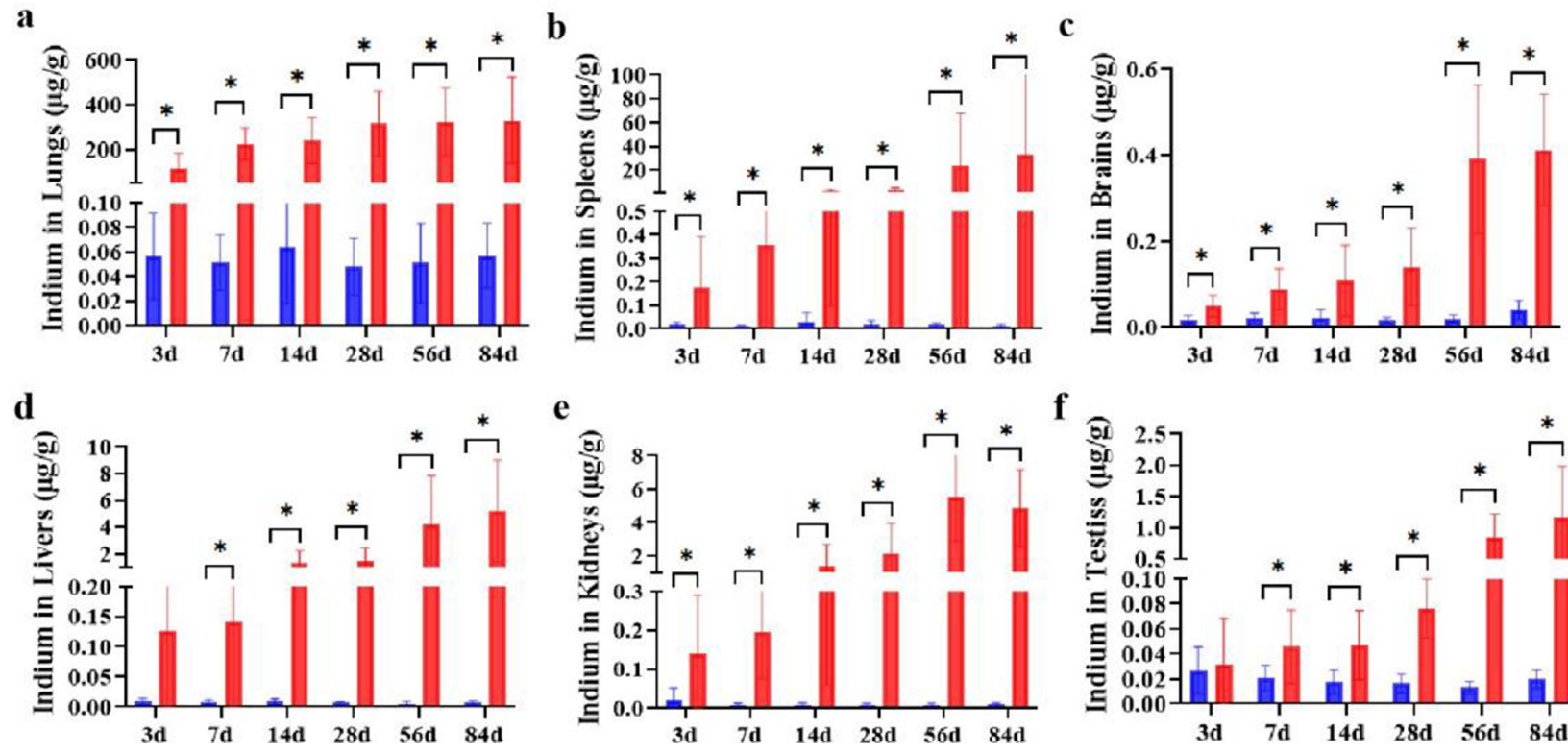

e
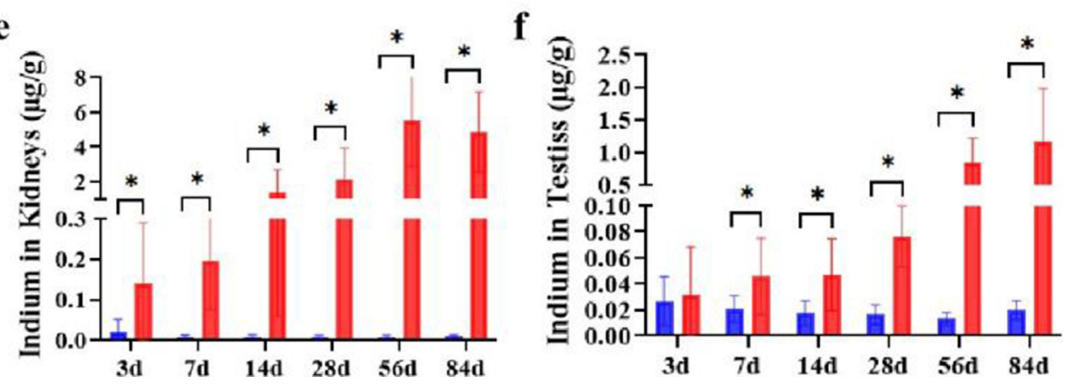

Figure 4 Indium concentration in organs. (A) The indium concentration of six tissues in dose group. The indium concentration of lungs (Ba), spleens (Bb), brains (Bc), livers (Bd), kidneys $(\mathbf{B e})$, and testes $(\mathbf{B f})$ in time group. $* P<0.00 \mathrm{I}$ vs control.

extremely slow after intratracheal instillation in rats. Indeed, between days 3 and 84, lung indium concentrations increased 3-fold in the $6 \mathrm{mg} / \mathrm{kg}$ Nano-ITO-exposed group. Interestingly, indium concentrations in the spleen and kidney of $6 \mathrm{mg} / \mathrm{kg}$ Nano-ITO-exposed rats displayed different kinetics when compared with lung indium, which peaked on day 56. Moreover, $6 \mathrm{mg} / \mathrm{kg}$ Nano-ITO-exposed rats at day 28 showed lung indium levels comparable with day 84 . One possible explanation is that the lung indium concentration reached saturation after 28 days of exposure.

\section{Hematological and Blood Biochemical Changes}

To assess exposure-induced systemic toxic effects after pulmonary Nano-ITO exposure, blood samples were analyzed for clinical and hematological parameters, in addition to biomarkers of metabolic, renal, and hepatic functions.

As Nano-ITO will remain in vivo for a prolonged period, changes in blood cells can reflect some pathological reactions induced by Nano-ITO. Routine blood analysis was performed and the results are shown in Figure 5. Most hematological parameters were similar between Nano-ITO-exposed rats and the control group. Compared with the control group, WBC counts, MCV levels, and PCL percentage were significantly altered in the Nano-ITO $3 \mathrm{mg} / \mathrm{kg}$ group 
A

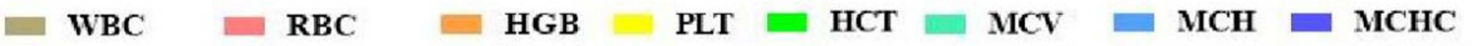

$$
\begin{aligned}
& \text { - RDW-SD RDW-CV } \square \text { PDW } \square \text { MPV } \square \text { PCL P-LCR } \square \text { NEUT } \square \text { LYMPH }
\end{aligned}
$$

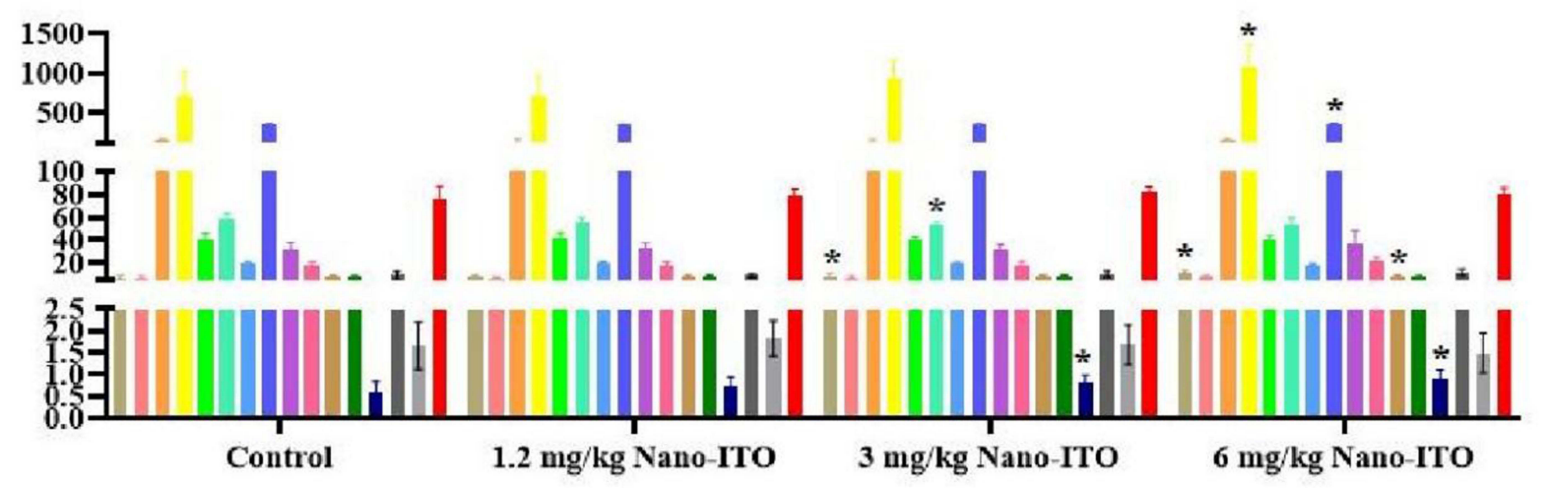

B

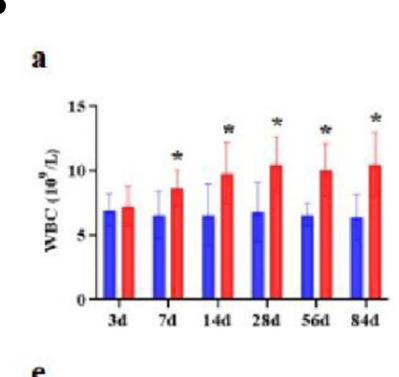

$\mathbf{e}$
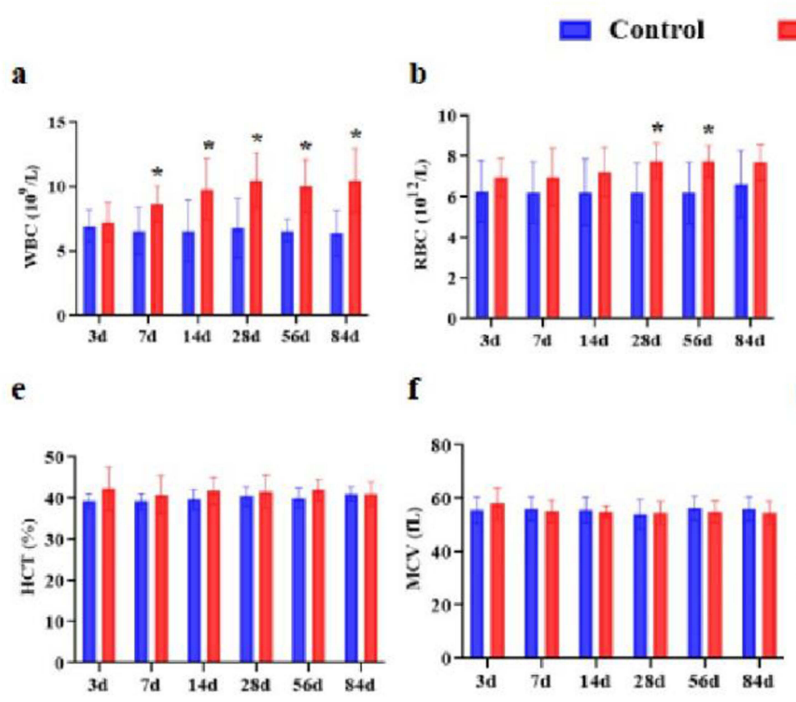

s

c $6 \mathrm{mg} / \mathrm{kg} \mathrm{Nano-ITO}$

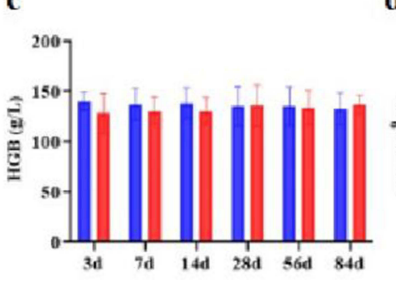

d
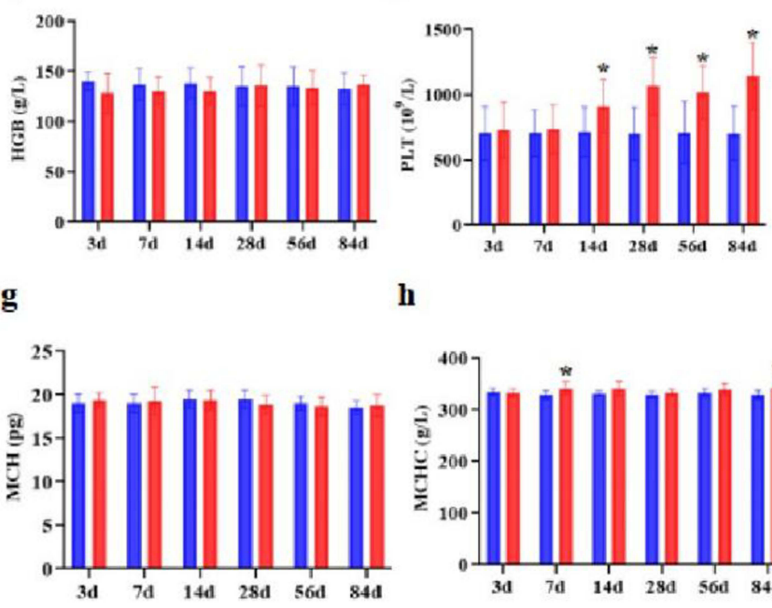

h

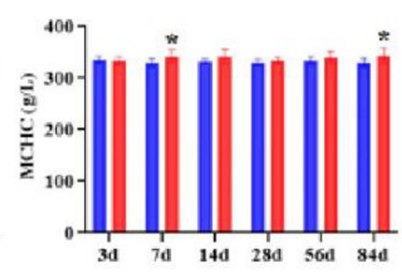

i

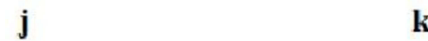

I
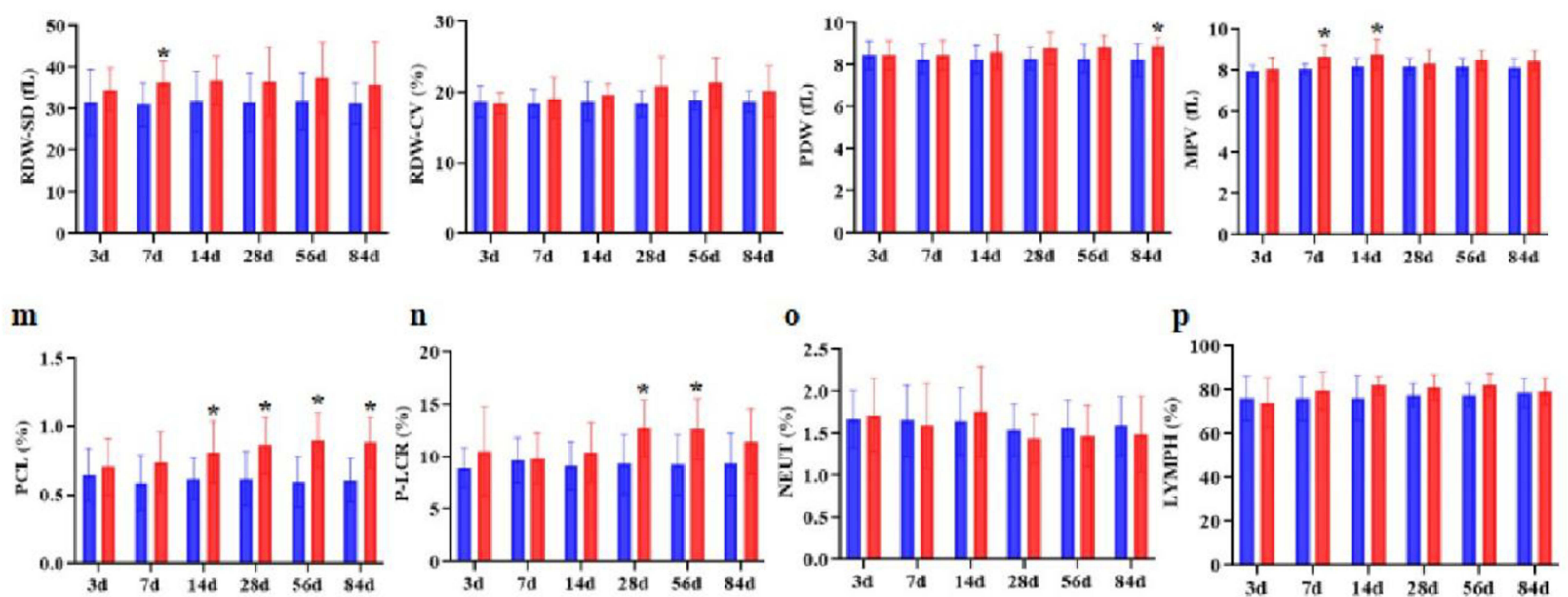

Figure 5 Hematology results of experimental rats. (A) Hematology results in dose group. (B) Hematology results in time group; (a) WBC levels; (b) RBC levels; (c) HGB levels; (d) PLT levels; (e) HCT levels; (f) MCV levels; (g) MCH levels; (h) MCHC levels; (i) RDW-SD levels; (j) RDW-CV levels; (k) PDW levels; (l) MPV levels; (m) PCL levels; (n) P-LCR levels; (o) NEUT levels; (P) LYMPH levels. ${ }^{*} P<0.05$ vs control. 
$(P<0.05)$. WBC and PLT count, MCHC and PDW levels, and PCL percentage were significantly higher in the NanoITO $6 \mathrm{mg} / \mathrm{kg}$ group than those in the control group $(P<0.05)$. The WBC counts, MCHC levels, RDW-SD, and MPV on day 7 were statistically higher in the Nano-ITO $6 \mathrm{mg} / \mathrm{kg}$ group than those in the control group $(P<0.05)$, thus indicating that Nano-ITO might cause an acute inflammatory response after entering the body. The WBC and PLT counts, MPV levels, and PCL percentage on day 14 were significantly higher in the Nano-ITO $6 \mathrm{mg} / \mathrm{kg}$ group than in the control group $(P<0.05)$. WBC, RBC, and PLT counts, PCL percentage, and P-LCR on day 28 and day 56 were remarkably higher in the Nano-ITO $6 \mathrm{mg} / \mathrm{kg}$ group than those in the control group $(P<0.05)$. WBC and PLT counts, MCHC levels, PCL percentage on day 84 were remarkably higher in the Nano-ITO $6 \mathrm{mg} / \mathrm{kg}$ group than those in the control group $(P<$ $0.05)$. No significant differences were detected in other parameters. The above results revealed that high-dose $(6 \mathrm{mg} / \mathrm{kg})$ Nano-ITO exposure can cause changes in hematological indexes. In particular, Nano-ITO could cause acute inflammation in the body, mainly inducing adverse effects on the WBC count and PCL.

Serum biochemistry tests were performed to determine the effects of Nano-ITO on the biological functions of major organs. Liver and renal function indexes including TP, ALB, GLB, A/G, ALT, AST, CHE, TBIL, DBIL, IBIL, BUN, UA, $\mathrm{Cr}$, MDA, SOD, HYP, CAT, GSH-px were measured and the results are presented in Figure 6A and B. Levels of TBIL and BUN were remarkably higher in the Nano-ITO $1.2 \mathrm{mg} / \mathrm{kg}$ group than in the control group $(P<0.05)$. TBIL, BUN and MDA were statistically higher in the Nano-ITO $3 \mathrm{mg} / \mathrm{kg}$ groups than in the control group $(P<0.05)$. AST, DBIL, BUN, UA, Cr, MDA, HYP, CAT, and, GSH-PX were significantly higher in the Nano-ITO $6 \mathrm{mg} / \mathrm{kg}$ groups than in the control group $(P<0.05)$. In high-dose Nano-ITO-exposed groups, levels of BUN, HYP, GSH-px on day 3, BUN and SOD on day 7, BUN, SOD, HYP, and GSH-px on day 14, GLB, DBIL, BUN, MDA, and GSH-px on day 28, A/G, IBIL, BUN, MDA, HYP, CAT, and GSH-px on day 56, AST, DBIL, BUN, UA, Cr, MDA, HYP, CAT, and GSH-px on day 84 were statistically higher than those estimated in the control group $(P<0.05)$. The results revealed that Nano-ITO could affect the liver and renal function of rats when exposed for 3 days, and Nano-ITO at high concentration induced serious effects, while low and middle concentrations showed relatively mild effects. Furthermore, renal function indexes including BUN, UA, and Cr, of all rats were detected, and the results are shown in Figure 6A and B. BUN levels were significantly altered in Nano-ITO-exposed rats when compared with the control group at the same sampling time, indicating that Nano-ITO at the examined doses induced kidney toxicity.

\section{Biochemical Analysis of BALF}

In the dose-response study, rats were exposed to $0,1.2,3$, and $6 \mathrm{mg} / \mathrm{kg}$ of Nano-ITO by intratracheal instillation and lavaged at week 12 post-exposure. The results of the biochemical analysis for the BALF supernatant are presented in Figure 7. SOD, T-AOC, MDA, TP, LDH, IL-1 $\beta$, IL-6, IL-10, and TNF- $\alpha$ levels were increased in all Nano-ITO-exposed groups. Although partly less apparent in the Nano-ITO $1.2 \mathrm{mg} / \mathrm{kg}$ group, levels were increased, except for altered SOD, showing a clear dose-dependent relationship. A statistically significant difference was observed in results for all groups, except for IL-1 $\beta$ and IL-10 in the Nano-ITO $1.2 \mathrm{mg} / \mathrm{kg}$ group when compared with those in the control group. In addition, the levels of MDA and TP in BALF showed a worsening pattern throughout the study period, with a good dosedependent relationship following repeated Nano-ITO exposure (Figure 7A). At week 12 post-instillation of $6 \mathrm{mg} / \mathrm{kg}$ of Nano-ITO, MDA was increased by more than 6-fold when compared with the control, while TP was enhanced by more than 40-fold in BALF, which could be attributed to severe cytotoxicity and increased vascular permeability of Nano-ITO.

As shown in Figure 7B, SOD, MDA, LDH activity, TP, and TNF- $\alpha$ levels were significantly increased in the BALF supernatant at all time points up to day 84 after intratracheal instillation in Nano-ITO groups when compared with corresponding controls. Although a slight decrease in SOD activity was observed in BALF of rats exposed to Nano-ITO for 14 and 28 days when compared with those exposed for 7 days, this decrease was not significant. T-AOC activity, IL$1 \beta$, IL-6, and IL-10 levels in BALF were measured to further examine pulmonary injury. We observed that cytokines measured in BALF show a proinflammatory response in the lungs of Nano-ITO-exposed rats. Significant differences in BALF levels of pro- or anti-inflammatory cytokines were detected at all examined time points. TNF- $\alpha$ was significantly elevated at all time points, whereas the anti-inflammatory cytokines IL-1 $\beta$, IL-6, and IL-10 were elevated at multiple time points. Slightly higher BALF levels of IL-6 were observed on day 3. IL-10 remained slightly increased at exposure day 14 , along with BALF levels of IL-1 $\beta$ at day 7 . The levels of these proteins, known for their protective role in lung 
A

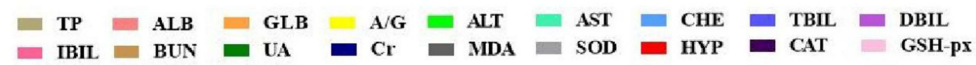

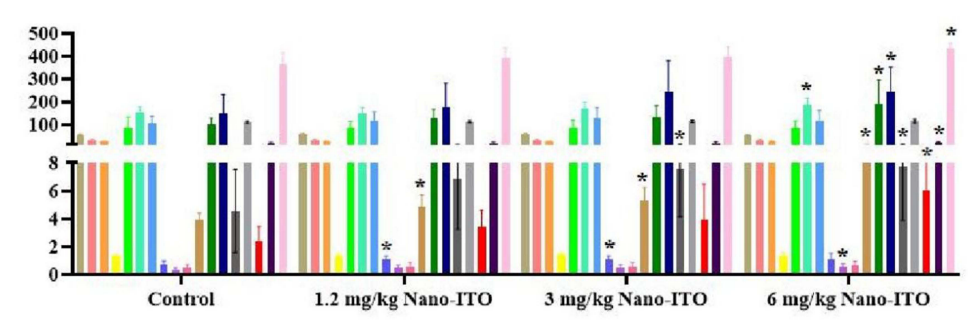

B

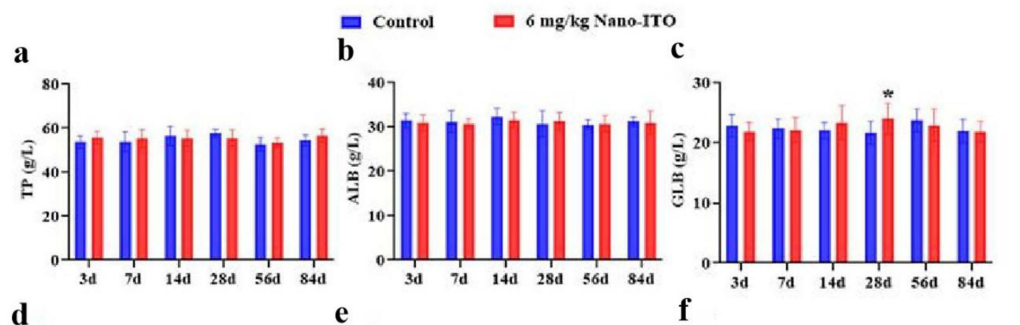

d

e

f

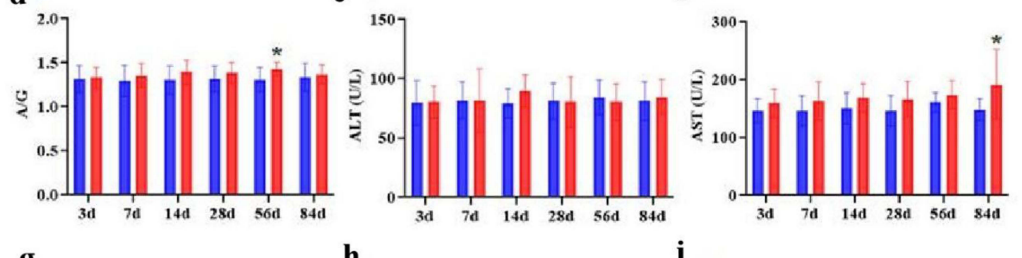

$\mathrm{g}_{250} \quad$ h ${ }_{2.0} \quad$ i
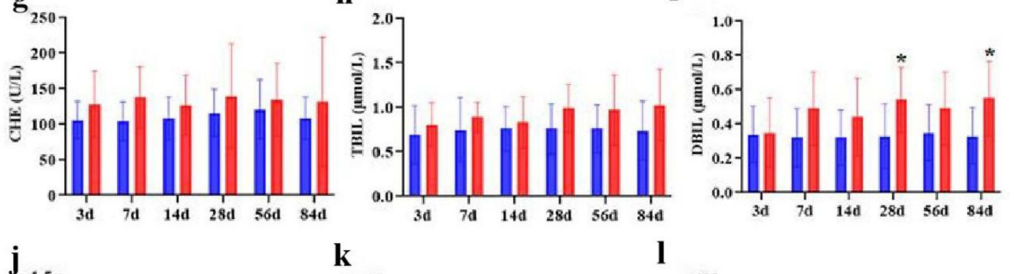

k
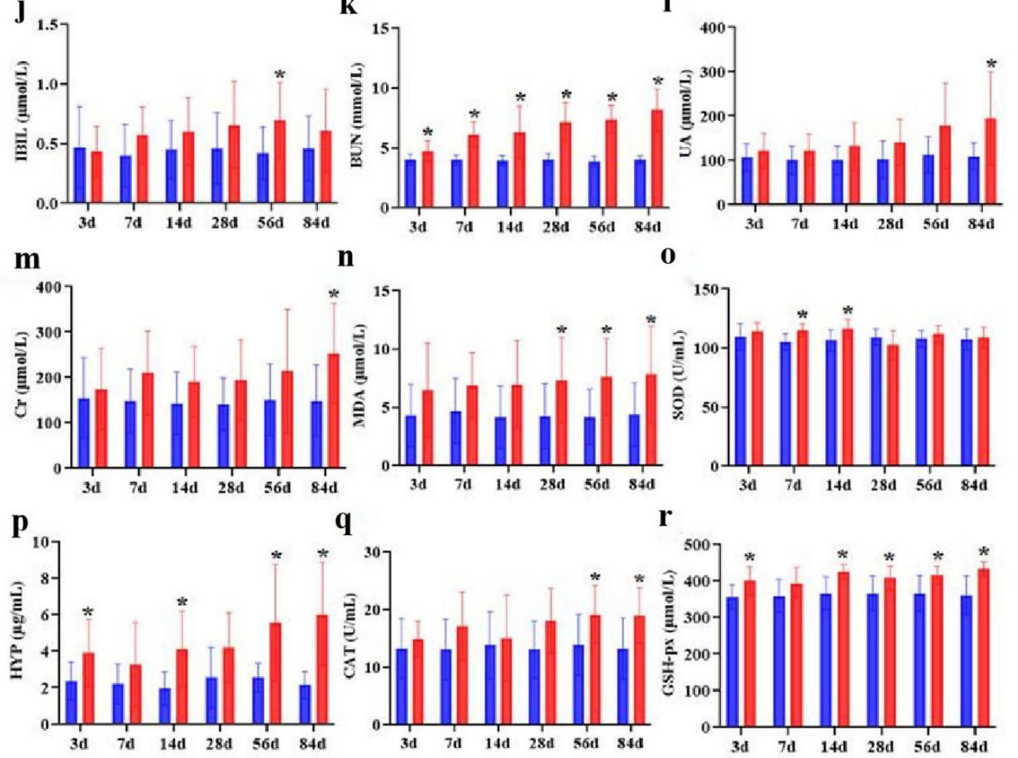

Figure 6 Serum biochemical results of experimental rats. (A) Serum biochemical results in dose group. (B) Serum biochemical results in time group; (a) TP levels; (b) ALB levels; (c) GLB levels; (d) A/G levels; (e) ALT levels; (f) AST levels; (g) CHE levels; (h) TBIL levels; (i) DBIL levels; (j) IBIL levels; (k) BUN levels; (l) UA levels; (m) Cr levels; (n) MDA levels; (o) SOD levels; (p) HYP levels; (q) CAT levels; (r) GSH-px levels. *P<0.05 vs control. 


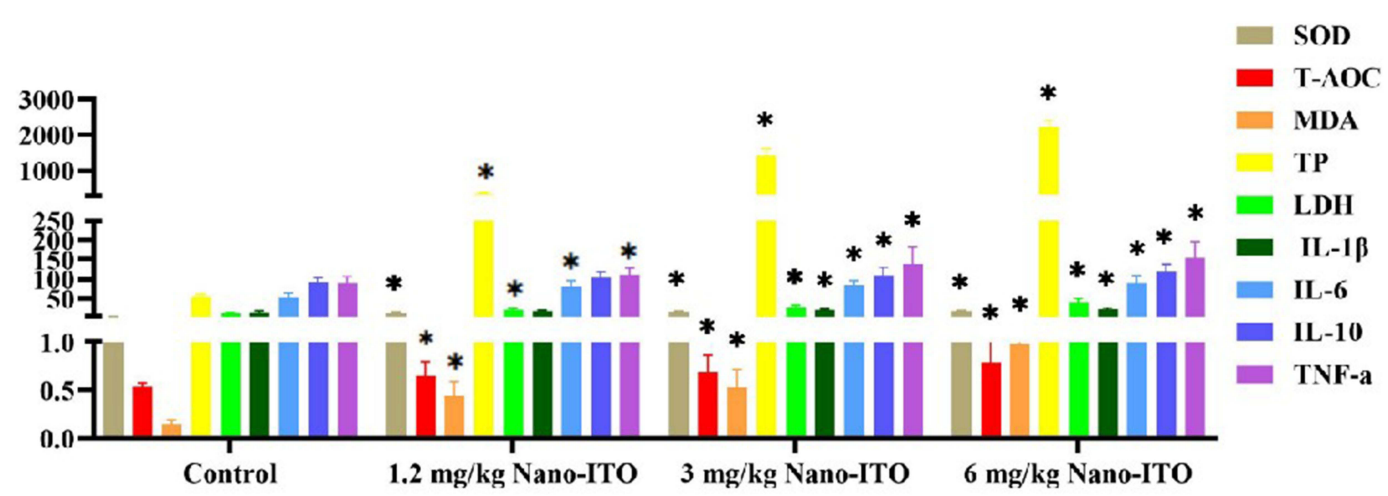

B

a

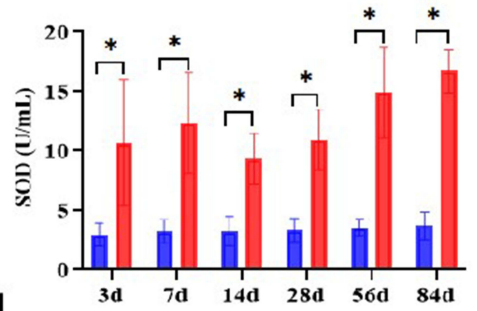

d

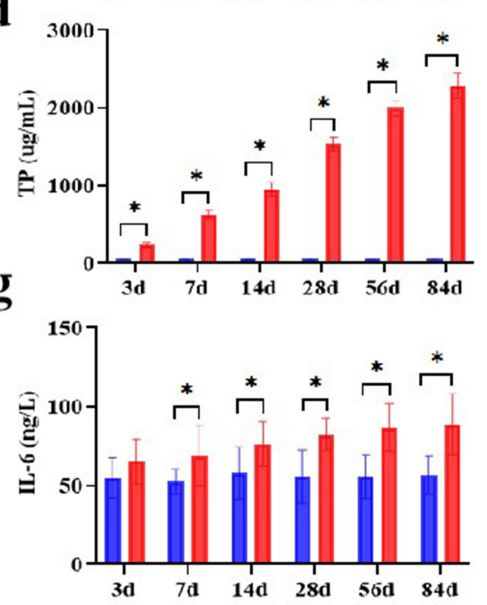

Control

b

e

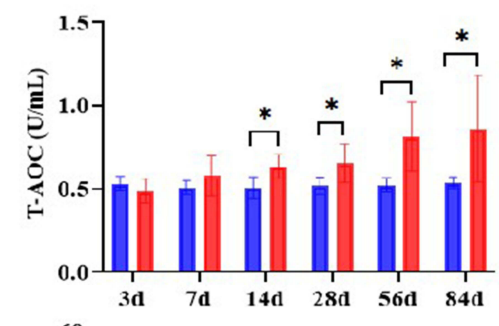

c
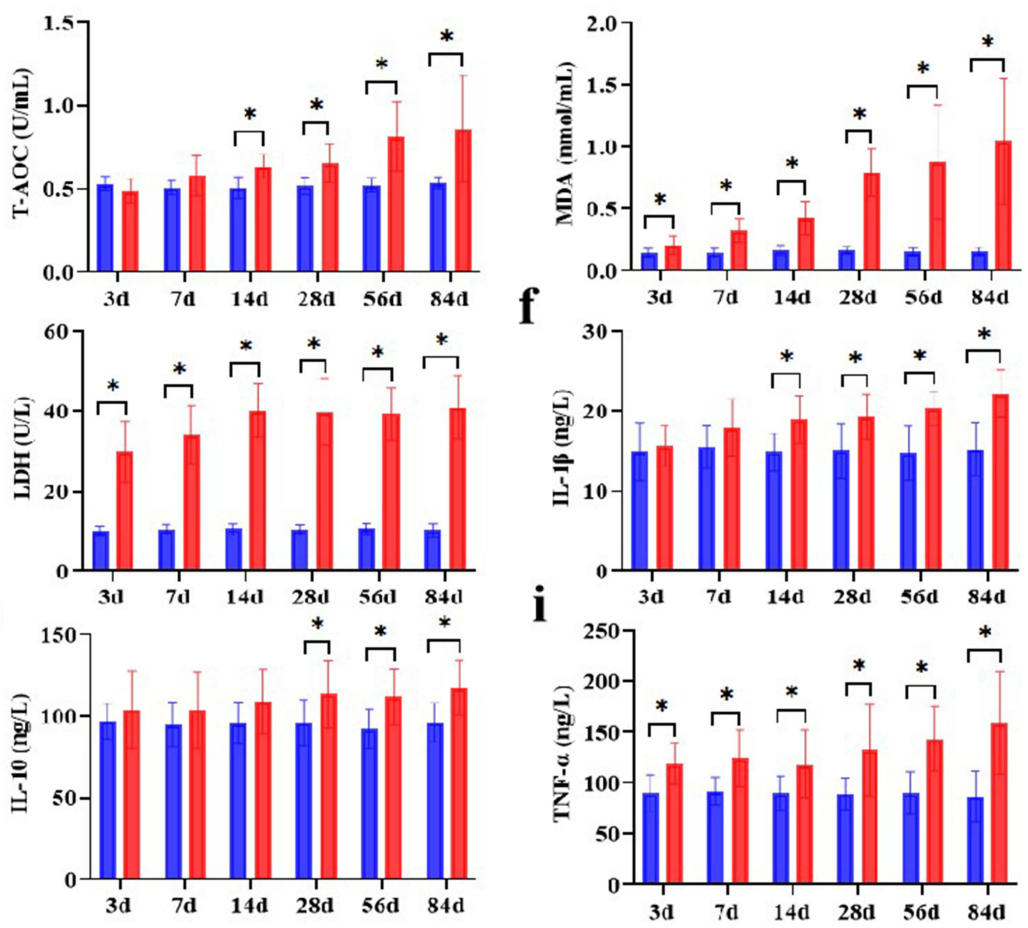

Figure 7 Analysis results of BALF. (A) BALF analysis results in dose group. (B) BALF analysis results in time group; (a) SOD levels; (b) T-AOC levels; (c) MDA levels; (d) TP levels; (e) LDH levels; (f) IL-I $\beta$ levels; (g) IL-6 levels; (h) IL- 0 levels; (i) TNF- $\alpha$ levels. Lung oxidative stress markers (SOD, T-AOC, and MDA); Pulmonary injury markers (TP, LDH); Inflammatory markers in airways (IL-I $\beta$, IL-6, IL-10, and TNF- $\alpha$ ). $* P<0.05$ vs control.

disease, were significantly increased in Nano-ITO-exposed rats, thus suggesting that their release could potentially alter the host's lung immune responses.

\section{Diverse and Progressive Histological Changes in the Lung}

To compare the histopathological changes in the lungs at all time points and different doses following Nano-ITO exposure, H\&E, PAS, Masson's trichrome, and Sirius red stained lung sections were microscopically examined for lung injury, inflammation, alveolar proteinosis, and interstitial fibrosis. In the control rat intratracheally instilled with physiological saline, normal lung parenchyma structure was observed, with mild lung inflammation. To observe the occurrence and development of PAP in Nano-ITO-exposed rats, three gradually increasing dosages $(1.2 \mathrm{mg} / \mathrm{kg}, 3 \mathrm{mg} / \mathrm{kg}$, 
$6 \mathrm{mg} / \mathrm{kg}$ ) for 12 weeks were employed and six distinct time points after $6 \mathrm{mg} / \mathrm{kg}$ Nano-ITO perfusion (Day 3, 7, 14, 28, $56,84)$ were accessed.

The representative histological lesions at week 12, exposed with different doses of Nano-ITO, are presented in Figure 8. The lungs showed dose-dependent damage. H\&E staining results of pulmonary pathological changes in all three Nano-ITO doses groups were almost identical morphologically, with the appearance of expanded alveolar spaces, pneumonia, and alveolitis was notably visible (Figure 8A). This lesion gradually worsened and was especially more marked in the Nano-ITO $3 \mathrm{mg} / \mathrm{kg}$ and $6 \mathrm{mg} / \mathrm{kg}$ groups when compared with the Nano-ITO $1.2 \mathrm{mg} / \mathrm{kg}$ group. In addition, $6 \mathrm{mg} / \mathrm{kg}$ Nano-ITO exposure induced extensive chronic pulmonary inflammation and lung interstitial fibrosis (seen as increased amounts of red stained fibrillar collagen), confirmed by Masson's trichrome staining and Sirius red staining, which showed an increase in collagen deposition and interstitial cell proliferation in the lung tissues, suggesting deposition of collagen in response to pulmonary injury and repair. Numerous enlarged and foamy macrophages were observed in the alveolar space, alveolar septa, and lumen of bronchi and bronchioles. An increased number of pulmonary intravascular macrophages was also observed. Nano-ITO exposure caused lung fibrosis, which was reflected by the thickening of alveolar septa and subepithelial areas of bronchi and bronchioles. The pleura was thickened owing to fibrosis. Masson's trichrome staining revealed a marked increase in fibrous tissue in the lung interstitium (Figure 8C). Furthermore, lymphocyte infiltration could be observed. Sirius red staining showed an increase in collagen deposition in the alveolar septa and peribronchial, peribronchiolar, perivascular areas, along with the proliferation of interstitial cells in the lung tissues (Figure 8D). In addition, focal chronic inflammation and interstitial fibrosis were observed in some areas of Nano-ITO $3 \mathrm{mg} / \mathrm{kg}$ groups, but to a considerably lesser extent than in the Nano-ITO $6 \mathrm{mg} / \mathrm{kg}$ groups. In contrast, no obvious, or only a mild fibrotic response, was observed in Nano-ITO $1.2 \mathrm{mg} / \mathrm{kg}$ group, although enlarged, particle-phagocytized macrophages could be detected in rat lungs. Most Nano-ITO particles were present in the cytoplasm of alveolar macrophages, with a few particles detected in alveolar spaces, interstitial tissue, and in the lymphoid tissue around bronchioles in the lung. These particles persisted in the lung up to the end of the observation period.

Additionally, PAP was observed in the $1.2 \mathrm{mg} / \mathrm{kg}, 3 \mathrm{mg} / \mathrm{kg}$ and $6 \mathrm{mg} / \mathrm{kg}$ Nano-ITO groups at 12 weeks post-exposure. Histopathological findings revealed that the alveoli, bronchi and bronchioli were filled with eosinophilic fine-granular fluid and cholesterol clefts (Figure 8B). The eosinophilic material stained positive with PAS. A moderately increased number of alveolar macrophages were detected, all with foamy cytoplasm. In addition, the alveolar walls were thickened. The severity of alveolar proteinosis and inflammatory cell infiltration in the lung increased in a dose-dependent manner. The Nano-ITO $6 \mathrm{mg} / \mathrm{kg}$ groups exhibited PAP, cholesterol crystals, alveolar fibrosis, pulmonary fibrosis, and alveolar emphysema. Furthermore, necrosis with inflammation, detachment of bronchial and bronchiolar epithelium, alveolar hemorrhage, and macrophages aggregation in alveolar or hilar lymph nodes were simultaneously observed in Nano-ITO $3 \mathrm{mg} / \mathrm{kg}$ and $6 \mathrm{mg} / \mathrm{kg}$ groups.

In brief, incidences of alveolar wall fibrosis, alveolar proteinosis, infiltration of alveolar macrophages and inflammatory cells, and alveolar epithelium hyperplasia were significantly increased in all Nano-ITO exposed groups (all three doses) at 12 weeks post-exposure. It is noteworthy that these histopathological changes accumulated with the Nano-ITO dose.
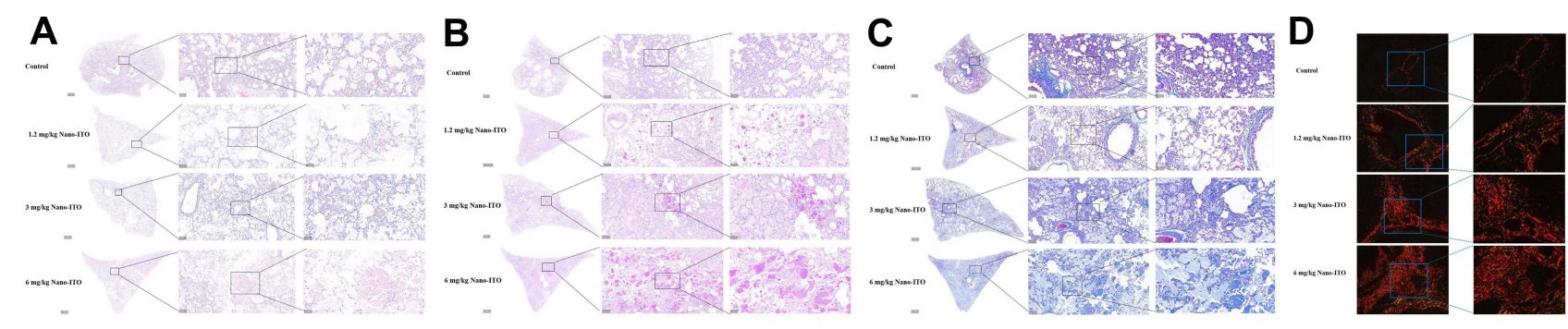

Figure 8 Microscopic findings of the serial lung tissue sections stained with H \& E (A), PAS (B), Masson's trichrome (C), and Sirius red (D) in dose-response study. Scale bar $=1000 \mu \mathrm{m}, 100 \mu \mathrm{m}$, and $50 \mu \mathrm{m}$ (the scale of each figure $A-C$ from left to right); Scale bar in figure $D$ represent $100 \mu \mathrm{m}$ and $50 \mu \mathrm{m}$ from left to right. 
The representative histological lesions at six time points following $6 \mathrm{mg} / \mathrm{kg}$ Nano-ITO are presented in Figure 9. Overall, repeated intratracheal Nano-ITO instillation resulted in progressive lung injury for up to 84 days. In control rats, intratracheally instilled with physiological saline, normal lung parenchymal structures were observed at the 6 corresponding time points. Time-dependent fibrosis, characterized by the formation of nodules, consisting of granuloma, alveolar epithelial hyperplasia, fibroblast proliferation with bundles of interlacing collagen fibers, progressive massive fibrosis, and diffuse interstitial fibrosis, was observed at all points following exposure in Nano-ITO groups. On day 3 post-exposure, we observed a large number of macrophages, admixed with few neutrophils, infiltrated into the alveolar walls, alveolar space, and interstitial tissues, along with focal alveolar epithelial cell hyperplasia and thickening of the alveolar septa (Figure 9A). The alveolar septum was broken, and a small amount of orange collagen was mixed in NanoITO particles (Figure 9D). On day 7 post-Nano-ITO exposure, minimal to mild septal fibrosis was observed; the alveolar contour was blurred and filled with numerous Nano-ITO particles of different sizes (Figure 9A). Type III collagen (light green) appeared outside the alveolar septum, which was thin and curved, showing a mesh rope shape (Figure 9D). Interestingly, the incidence of interstitial fibrosis and inflammatory cell infiltration into the alveolar space and interstitial tissues was significantly increased following exposure to $6 \mathrm{mg} / \mathrm{kg}$ Nano-ITO at 14 days (Figure 9A). The normal alveolar structure disappeared, and instead, two kinds of collagen appeared, the thicker type I collagen (orange and dark red) and the thinner, light green (Figure 9D). On day 28 post-exposure, bronchiolization of the alveoli, characterized by the replacement of alveolar epithelial cells with bronchiolar-type epithelium and emphysematous changes, was observed in the thickened alveolar septa. Other microscopic changes included prominent type II pneumocyte hypertrophy and hyperplasia, pleural inflammation and fibrosis, and variable amounts of alveolar exudation consistent with PAP (Figure 9A and B). Diffuse, light green, curved collagen existed in the form of a mesh mixed with a small amount of thick orange fiber and numerous Nano-ITO particles (Figure 9D). Fifty-six days post-exposure, the occurrence of septal fibrosis was similar across groups; light green collagen was further increased, while orange collagen was relatively less (Figure 9A-D). Furthermore, microscopic findings such as massive fibrotic lesions in the lung tissue at 84 days after Nano-ITO exposure, showing hyperplasia of type II epithelial cells along with the frequent appearance of spindle and elongated shape, was found to be associated with the presence of macrophages loaded with high particle content, suggesting a correlation between lung tissue damage and the presence of particles (Figure 9A). The two types of collagen diffusely filled the whole field of vision, and both the thick orange collagen and thin light green collagen increased significantly; they were interlaced in a mesh shape, with numerous Nano-ITO particles (Figure 9D). The Nano-ITOexposed lungs showed higher collagen accumulation than those of the control group, as measured by Masson's trichrome staining, with lung sections exhibiting an increasing amount of blue stain with time, thus indicating connective tissue accumulation (mainly collagen deposition), becoming more intense at the later time points (Figure 9C). The above data collectively indicate that Nano-ITO can accelerate lung fibrosis in SD rat.

The severity of fibrotic and inflammatory changes in the lung ranged from minimal to severe depending on the exposure time. Notably, PAP occurred on day 28 , tended to be more severe on days 56 and 84 , and its severity was
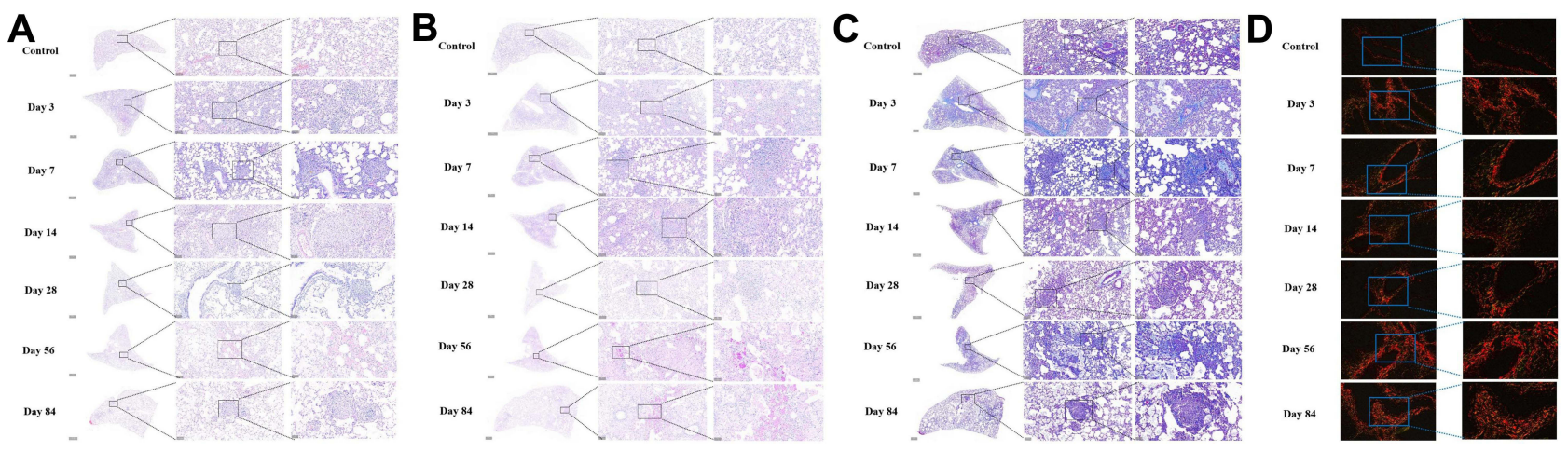

Figure 9 Microscopic findings of the serial lung tissue sections stained with H \& E (A), PAS (B), Masson's trichrome (C), and Sirius red (D) in time-course study. Scale bar = $1000 \mu \mathrm{m}, 100 \mu \mathrm{m}$, and $50 \mu \mathrm{m}$ (the scale of each figure A-C from left to right); Scale bar in figure D represent $100 \mu \mathrm{m}$ and $50 \mu \mathrm{m}$ from left to right. 
gradually enhanced with elapsing time after the intratracheal instillation, although the majority of these rats showed fibrotic pathology. For example, neutrophil infiltration in alveoli was observed from day 3, foamy macrophages were infiltrated from day 14, proteinous materials were deposited from day 28, and cholesterol cleft deposition was observed from day 56 in the $6 \mathrm{mg} / \mathrm{kg}$ Nano-ITO group. Histological examinations revealed lymphocyte infiltration into the alveolar walls and eosinophilic surfactant material and macrophages in the alveolar lumina (Figure 9B). The infiltration of proteinous materials, cholesterol clefts, foamy macrophages, and neutrophils in the alveolar region was notably evident. In the interstitial region, infiltration of inflammatory cells in the peribronchial and perivascular region, type II cell hyperplasia, and the formation of granulomatous inflammation with giant cells were observed from day 28. Although lung fibrosis appeared and progressed during the disease course, PAP gradually worsened at the late stage (56, 84 days), indicating that alveolar proteinosis tended to occur after day 56 of repeated Nano-ITO exposure. Overall, the pathology was most severe in the lungs of rats exposed for 84 days. The induction time-point of these parameters in the alveolar region was consistent with BALF analysis data.

\section{Histopathology of Spleen, Brain, Liver, Kidney and Testis}

Histological assessment of secondary organs was performed to evaluate tissue damage and inflammation caused by Nano-ITO exposure. The main organs of all rats were sliced into 5- $\mu \mathrm{m}$ sections and stained with H\&E. Histopathological changes were observed in the spleen, brain, liver, kidney, and testis of male rats treated with Nano-ITO at different treated doses and for distinct times. The representative histological results are shown in Figure 10. Following the instillation of Nano-ITO at $6 \mathrm{mg} / \mathrm{kg}$ body weight, we observed a marked inflammatory response or pathological changes in the liver, kidney, and testis of all rats at different sampling times when compared with the control group. Nano-ITOexposed rats showed slight edema in some liver cells, mild inflammatory cell infiltration and dilated sinusoids in the liver (Figure 10), a mild-to-moderate degree of inflammatory cell infiltration, slightly vacuolar degeneration in renal tubular epithelial cells, hyaline cast/cell debris in tubules, dilated tubules, and glomerular atrophy in the kidneys. The incidence and severity of these findings were higher in the $6 \mathrm{mg} / \mathrm{kg}$ Nano-ITO-exposed rats than those in $1.2 \mathrm{mg} / \mathrm{kg} \mathrm{or} 3 \mathrm{mg} / \mathrm{kg}$ exposed rats. Red foci and decreased size in the liver and testes were observed in rats exposed to $6 \mathrm{mg} / \mathrm{kg}$ Nano-ITO; additionally, some degenerative vacuolar changes were detected in both interstitial and seminiferous tubules. Other organs, such as the brain, showed no pathological changes (Figure 10). Therefore, Nano-ITO could accumulate and persist in the organs of rats, especially in the liver, kidney, and testis, exhibiting obvious histopathological abnormalities, which suggested that Nano-ITO caused toxicity to these organs at different doses.

\section{Discussion}

The present study was inspired by accumulating evidence of "indium lung disease" owing to workplace inhalation exposure to indium and its compounds, characterized by PAP, fibrosis, cholesterol clefts, and granulomas. ${ }^{17}$ To date, it has been reported that the occurrence and development of various lung diseases such as pulmonary fibrosis, emphysema, and PAP in indium occupational workers significantly correlate with exposure levels to indium compounds. ${ }^{1,2,6,17,18}$ However, limited data are available in terms of ITO-induced pulmonary and systemic toxicities. There exists a considerable demand for ITO in consumer electronics ${ }^{19}$ manufacture, typically produced in the nanoscale range. However, biodistribution, toxicological properties, and underlying mechanism(s), both direct or indirect, of indium have not been sufficiently investigated.

Body weight and organ weight/body weight coefficients are two important indicators for the comprehensive toxicological assessment of experimental animals exposed to occupational hazardous substances. The present study demonstrated that repeated intratracheal instillation of Nano-ITO into the lungs significantly induced extensive toxicity in male SD rats without decreasing body weight. The lung was the main target organ for indium accumulation and toxicity, and significant dose-dependent changes in lung appearance, relative weight, and histopathology were observed throughout the post-exposure periods. Based on our findings, the lung indium concentration reached saturation after 28 days of exposure, which was comparable with those at days 56 and 84. Indium accumulation in the secondary organs occurred in the following order: spleen $>$ liver $>$ kidney $>$ testis $>$ brain. Significant histopathological changes could be detected in extra-pulmonary organs, such as the liver, kidney, and testis; this induced Nano-ITO migration from the lungs to the 


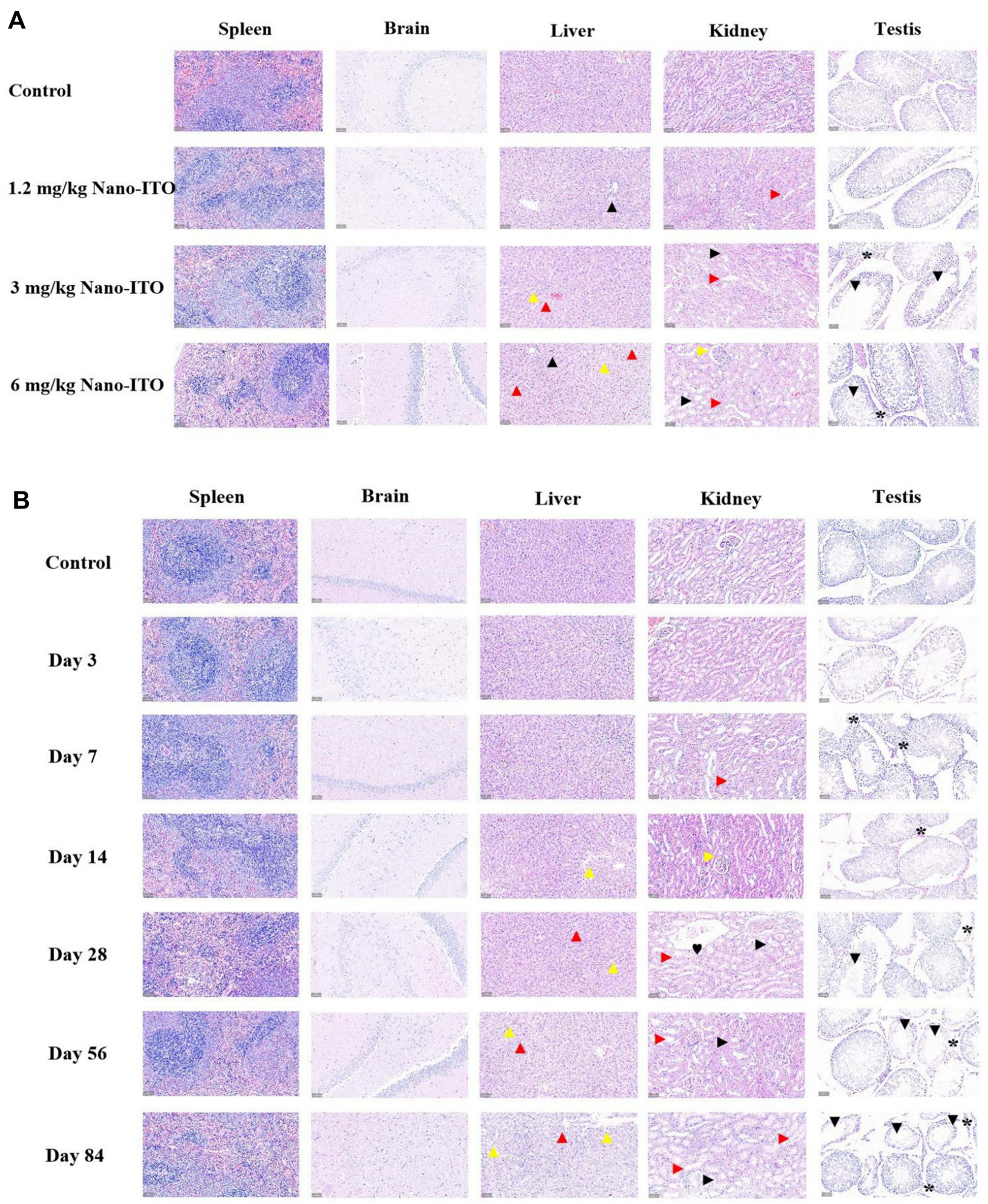

Figure 10 Representative histological images of major organs including spleen, brain, liver, kidney and testis stained with hematoxylin and eosin stain ( $\mathrm{H} \& \mathrm{E})$. Scale bar $=50$ $\mu \mathrm{m}$. Liver: ${ }^{\boldsymbol{\Delta}}$ Mild inflammatory cell infiltration, ${ }^{\boldsymbol{\Delta}}$ Vacuolation, and ${ }^{\boldsymbol{\Delta}}$ Sinusoid dilation. Kidney: $\boldsymbol{}^{-}$Vacuolar degeneration in renal tubular epithelial cells, $\boldsymbol{}^{-}$Dilated tubules, Atrophy of glomeruli, and ${ }^{\vee}$ Cell debris in tubules. Testis: ${ }^{\nabla}$ Vacuolization of seminiferous tubules, $\star V$ acuolization of interstitial cells. 
secondary organs, significantly and steadily increasing the indium retention burden in those organs. However, the indium levels in the liver and kidney were significantly higher than those in other extra-pulmonary organs of Nano-ITO exposed rats. The higher indium accumulation in the liver and kidney impaired their function. Simultaneously, serum biochemistry results revealed that Nano-ITO affected the liver and renal function of rats exposed for 12 weeks. In addition, the highest Nano-ITO dose induced the most serious effect, whereas the effects of Nano-ITO at low and middle concentrations were relatively mild. Thereby, the injured liver and kidney further hindered the removal of indium from the body, resulting in increased indium accumulation in the extra-pulmonary organs. Although indium retention in extrapulmonary organs was low, indium accumulation in these organs indicated that translocation from the lungs occurred during the post-exposure period.

Clinical cases of indium-induced PAP, namely, indium lung disease, are mostly occupationally mediated. However, regarding Nano-ITO-induced pulmonary fibrosis in animal models, a consensus is yet to be reached. In the present study, we showed that intratracheal instillation of Nano-ITO produced various pathological changes in rats when compared with those observed in human case reports including PAP, cholesterol clefts in the alveoli, foamy macrophages, type II cell hyperplasia, fibrosis, and granulomas, ${ }^{17}$ worsening in a time- and dose-dependent manner. Furthermore, using histopathological analysis of H\&E staining, PAS stain, Masson's trichrome, and Sirius red staining, we found that Nano-ITO accelerated alveolar proteinosis and lung fibrosis formation with granulomatous inflammation in male SD rats. Interestingly, acute inflammatory reactions were induced from day 3, while pulmonary fibrosis occurred from day 7 and worsened throughout the study. PAP occurred on day 28 , tended to be more severe on days 56 and 84, and its severity was gradually enhanced with elapsing time after the intratracheal instillation, although most rats exhibited fibrotic pathology. This onset of PAP is contrasted with the previous animal studies, which only required two weeks of repeated inhalation or instillation exposure to induce PAP. ${ }^{20,21}$ For example, Jeong et al have shown that PAP was induced from day 3 and fully developed at 2 weeks. ${ }^{22}$ The higher protein deposition responses in alveoli and earlier onset of pulmonary fibrosis observed in the present study, compared with previous studies, could be attributed to the higher deposition of indium nanoparticles in the alveoli than indium compounds. Therefore, the novelty of this study is that we evaluated the dose- and time-dependent lung injuries induced by Nano-ITO more accurately over a longer time frame.

Considering histopathological results, the increased lung weight was attributed to the presence of PAP, as the occurrence of PAP for each dose was consistent with the changes in lung weight, and this lesion caused an abnormal accumulation of lipoproteinaceous material within the alveoli. In this context, the BALF exhibited a turbid, milky appearance, and abnormal alveolar macrophages were observed in the BALF of various doses and at multiple time points. It is well-known that milky fluid and foamy alveolar macrophages are commonly observed in the BALF of patients with PAP. Biochemical analysis of BALF can be used to evaluate Nano-ITO-induced alterations in pulmonary cell damage, inflammatory cell infiltration, cytokine release, and surface release of cellular secretory products. For example, LDH is a cytoplasmic enzyme extracellularly released when cells are damaged. The enzyme activity in BALF could provide a quantitative assessment of cell and tissue damage following lung exposure to Nano-ITO. Accumulation of neutrophils and macrophages in the lungs are also key events in the inflammatory response following intratracheal Nano-ITO instillation. Our results showed that exposure to Nano-ITO leads to severe acute lung injury and lung inflammation, which is reflected by increased LDH activity in BALF, confirmed by histopathological examination. These effects of Nano-ITO were dose-related and were most severe at 3 and $6 \mathrm{mg} / \mathrm{kg}$ Nano-ITO exposure. Thus, we selected a dose of $6 \mathrm{mg} / \mathrm{kg}$ Nano-ITO for exposing rats in the following comparative studies.

To evaluate Nano-ITO-induced inflammatory responses in alveoli, a panel of pro- and anti-inflammatory cytokines were measured in BALF. The BALF levels of the inflammatory mediators, IL-1 $\beta$, IL- 6 , and TNF- $\alpha$, showed dose and timedependent increases, consistent with the data indicating progressive lung damage induced by Nano-ITO. The increased IL-1 $\beta$ implies that Nano-ITO can activate the NALP3 inflammasome, consistent with the previous studies using different indium compounds such as sintered ITO ${ }^{13}$ and ITO. ${ }^{23}$ The activation of the NLRP3 inflammasome by Nano-ITO can induce progressive lung injuries and persistent neutrophilic inflammation. Along with inflammation, oxidative stress is a key mechanism linking Nano-ITO exposure to detectable physiological and biochemical responses. As such, SOD, T-AOC, and MDA levels were measured to determine the extent of protein and lipid oxidation after exposure to Nano-ITO (Figure 9). Regarding oxidative stress analysis, the oxidative stress produced by Nano-ITO in the BALF can increase the activity of 
relevant mediators, which can stimulate the production of proinflammatory cytokines, as observed in the present study. Simultaneously, a disturbance of cytokines secreted into BALF reflects immunological reactions of the lung in response to Nano-ITO. Nano-ITO exposure significantly increased TP and LDH, which are cell damage indicators, in the BALF. In addition, histopathological examination revealed that the rat lungs showed alveolar proteinosis at 56 days post-exposure; in contrast, the lungs at 3,7,14 days post-dose were negative for alveolar proteinosis. Meanwhile, IL-10 was significantly increased in BALF at 28, 56 and 84 days post-exposure when compared with that at 3, 7, 14 days post-exposure. Therefore, IL-10 of BALF seems to be related to alveolar proteinosis. At days 3 and 7 of exposure, we simultaneously noted a slight increase in TNF- $\alpha$, a cell-signaling cytokine produced by activated macrophages and involved in acute systemic inflammation, and IL-6, a cytokine that exerts both proinflammatory and anti-inflammatory functions. All findings suggested that in connection with the infiltration of inflammatory cells and PAP, proinflammatory cytokines (IL-1 $\beta$, IL-6, IL-10, TNF- $\alpha$ ), cellular damage indicators (TP, LDH), and oxidative stress markers (SOD, T-AOC, MDA) were markedly increased in BALF obtained from all Nano-ITO groups after the 28-day exposure period. These changes worsened in the $6 \mathrm{mg} / \mathrm{kg}$ Nano-ITO group at 28 days post-exposure. It was speculated that the series of prolonged and deteriorated changes could be attributed to the slow elimination of these materials in the $6 \mathrm{mg} / \mathrm{kg}$ group, resulting in apparent cell damage and alveolar macrophage impairment in the lung. The possible mechanism underlying lung damage was not determined in the present study. However, there is a strong possibility that oxidative stress and inflammatory reaction played a role in mediating lung damage.

Increased platelet count is a notable finding as it is also associated with cardiovascular effects, suggesting a possible link between Nano-ITO and cardiovascular events. We also observed an interesting phenomenon, such that Nano-ITO could cause acute inflammation in the body, adversely impacting the WBC count and PCL. It is known that collagen hyperplasia-induced myelofibrosis seriously affects hematopoietic function. Next, we plan to examine the influence of Nano-ITO-induced pulmonary fibrosis on the hematopoietic potential of the lungs in SD rats to establish a more comprehensive understanding of the impact of Nano-ITO on the human body.

Interestingly, some of these disorders reported in the indium-exposed workers, including inflammation and granulomas, were also observed in the lungs of male rats exposed to Nano-ITO by intratracheal instillation in the present study. Differences in the routes of indium exposure, its dose and duration of exposure between male rats used in the present study and indium-exposed workers need to be considered, in addition to a distinct species difference between rats and humans. However, researchers have demonstrated that the relative lung toxicity potentials of particles could be appropriately evaluated using intratracheal or inhalation exposures. Therefore, the present findings of toxic pulmonary responses in male rats exposed to intratracheal Nano-ITO instillation provide novel information regarding the hazards of lung toxicity. It should be highlighted that the Nano-ITO dose (6 mg/kg rat) used in the present study appears high and may cause lung tissue overloading when compared with the estimated inhalation exposure to indium compound particles in workers under occupational settings. Further studies with lower doses of Nano-ITO will be needed to delineate the dose-response relationships, including the no observed effect level for pulmonary toxic responses.

In summary, based on the data from the present study, we arrived at the following conclusion regarding the consequences of Nano-ITO-exposure in rat lungs (Figure 11). Nano-ITO intratracheal instillation caused pulmonary toxicity by inducing acute inflammation at 3 days, pulmonary fibrosis at 14 days, and alveolar proteinosis at 56 days postexposure. During acute inflammation, Nano-ITO-induced bronchial epithelial cells underwent injury and apoptosis caused by ROS generation, inflammatory cell infiltration, and the secretion of proinflammatory cytokines. During the fibrosis period, Nano-ITO induced type II cell hyperplasia, intra-alveolar fibrotic changes, alveolar septum thickening, promoted pulmonary fibrosis caused by myofibroblast activation, and resulted in collagen deposition, along with the notable formation of granulomatous inflammation with giant cells. During alveolar proteinosis, neutrophil infiltration in alveoli was observed first, followed by foamy macrophages; subsequently, PAP was found to occur, followed by deposition of cholesterol clefts.

\section{Conclusion}

Indium lung disease is a worldwide concern. The findings of the present study suggest that repeated intratracheal NanoITO instillation can worsen lung damage manifested as acute inflammatory reactions, pulmonary fibrosis, PAP, cholesterol clefts in the alveoli, foamy macrophages, type II cell hyperplasia, and granulomas. Accordingly, we successfully 


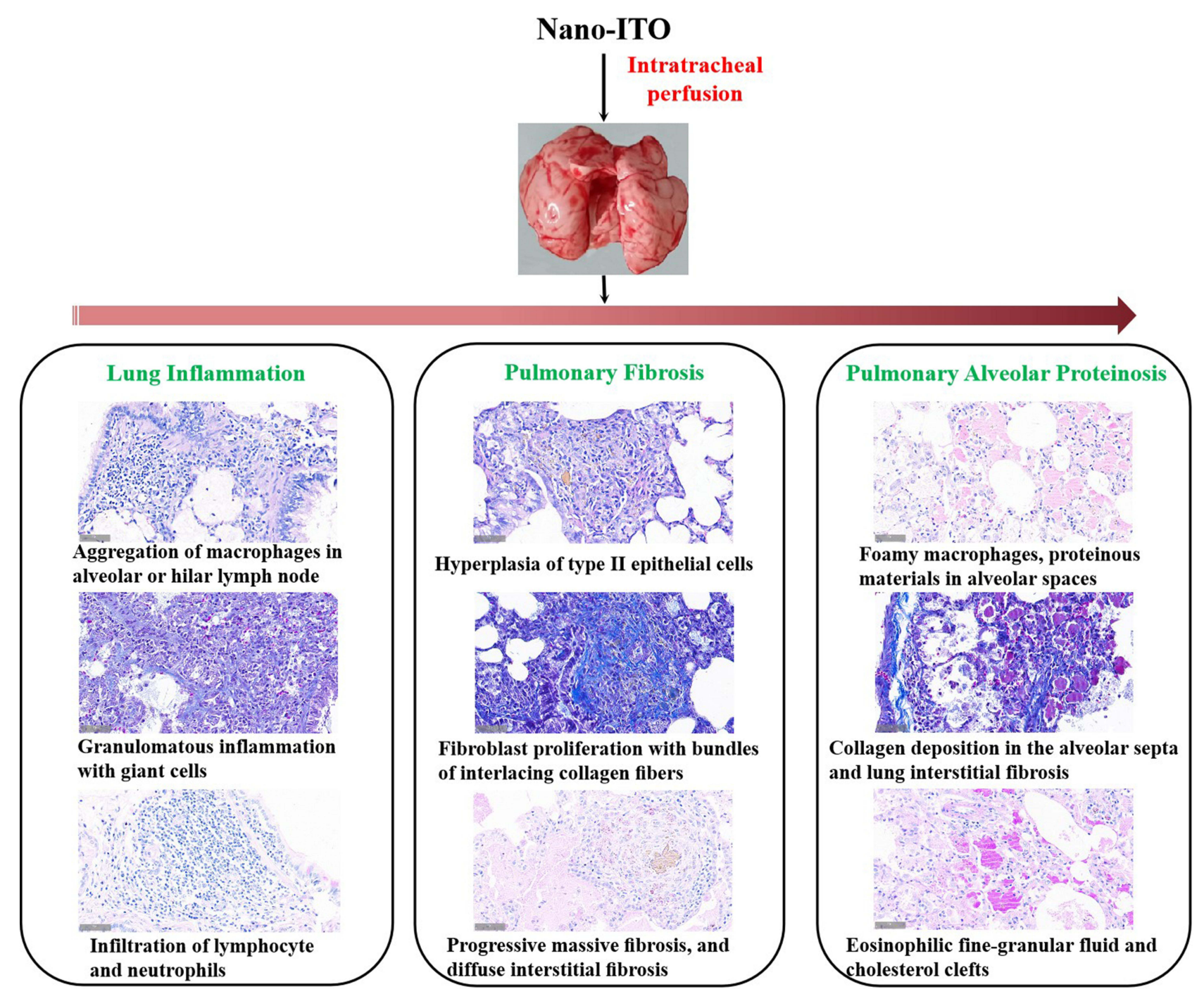

Figure II Schematic depiction of the mechanism of Nano-ITO involved in pulmonary inflammation, fibrosis, and PAP events.

reported the systemic toxicological effects following repeated exposure to Nano-ITO. The repeated administration of different doses, instead of a single dose, and 6 distinct sampling time points, enable an approximation to their real exposure conditions, often during occupational exposure and on a subacute to chronic basis. In vivo studies demonstrated that repeated intratracheal Nano-ITO instillation in male SD rats primarily induced lung toxicity via induction of acute inflammation at 3 days, pulmonary fibrosis at 14 days, and alveolar proteinosis at 56 days post-exposure, corroborating mechanisms underlying pathologies observed in animal disease models related to indium occupational exposure. Indeed, to the best of our knowledge, this is the first study comparatively focusing on pulmonary and systemic toxicity following consecutive exposure of Nano-ITO. Herein, we reported the occurrence of PAP associated with progressive fibrosis, the pathological features of the lung, and our assessment regarding the process of fibrosis formation. We believe our result is not only positioned to provide a springboard for other investigators to explore the role of Nano-ITO in the respiratory system but also draws attention to this rapidly evolving area of human exposure-induced diseases.

\section{Acknowledgments}

The authors gratefully acknowledge the technical assistance provided by Li Bin at the Institute for Occupational Health and Poison Control, China Center for Disease Prevention and Control. All authors declare that there are no conflicts of interest regarding the publication of this article. 


\section{Disclosure}

The authors report no conflicts of interest in this work.

\section{References}

1. Homma S, Miyamoto A, Sakamoto S, et al. Pulmonary fibrosis in an individual occupationally exposed to inhaled indium-tin oxide. Eur Respir J. 2005;25(1):200-204. doi:10.1183/09031936.04.10012704

2. Chonan T, Taguchi O, Omae K. Interstitial pulmonary disorders in indium-processing workers. Eur Respir J. 2007;29:317-324. doi:10.1183/ 09031936.00020306

3. Cummings KJ, Donat WE, Ettensohn DB, et al. Pulmonary alveolar proteinosis in workers at an indium processing facility. Am J Respir Crit Care Med. 2010;181(5):458-464. doi:10.1164/rccm.200907-1022CR

4. Xiao YL, Cai HR, Wang YH, et al. Pulmonary alveolar proteinosis in an indium-processing worker. Chin Med J. 2010;123(10):1347-1350.

5. Omae K, Nakano M, Tanaka A, et al. Indium lung-case reports and epidemiology. Int Arch Occup Environ Health. 2011;84(5):471-477. doi:10.1007/s00420-010-0575-6

6. Amata A, Chonan T, Omae K, Nodera H, Terada J, Tatsumi K. High levels of indium exposure relate to progressive emphysematous changes: a 9-year longitudinal surveillance of indium workers. Thorax. 2015;70:1040-1046. doi:10.1136/thoraxjnl-2014-206380

7. Watanabe Y, Kawabata Y, Koyama N, et al. A clinicopathological study of surgically resected lung cancer in patients with usual interstitial pneumonia. Respir Med. 2017;129:158-163. doi:10.1016/j.rmed.2017.06.015

8. Liu N, Guan Y, Xue L, et al. Assessment of DNA/chromosome damage in the peripheral blood lymphocytes of workers exposed to indium compounds. Toxicol Sci. 2017;157(1):41-49. doi:10.1093/toxsci/kfx017

9. Tsao YC, Fan HY, John Luo JC. Case reports of indium lung disease in Taiwan. J Formosan Med Assoc. 2021;120:893-898. doi:10.1016/j. jfma.2020.08.009

10. Tanaka A, Hirata M, Homma T, Kiyohara Y. Chronic pulmonary toxicity study of indium-tin oxide and indium oxide following intratracheal instillations into the lungs of hamsters. J Occup Health. 2010;52:14-22. doi:10.1539/joh.L9097

11. Gwinn WM, Qu W, Shines CJ, et al. Macrophage solubilization and cytotoxicity of indium-containing particles in vitro. Toxicol Sci. 2013;135:414-424. doi:10.1093/toxsci/kft154

12. Lim CH, Han JH, Cho HW, Kang M. Studies on the toxicity and distribution of indium compounds according to particle size in Sprague-Dawley rats. Toxicol Res. 2014;30:55-63. doi:10.5487/tr.2014.30.1.055

13. Badding MA, Schwegler-Berry D, Park JH, Fix NR, Cummings KJ, Leonard SS. Sintered indium-tin oxide particles induce pro-inflammatory responses in vitro, in part through inflammasome activation. PLoS One. 2015;10:e0124368. doi:10.1371/journal.pone.0124368

14. Baddinga MA, Fixa NR, Orandlea MS, et al. Pulmonary toxicity of indium-tin oxide production facility particles in rats. $J$ Appl Toxicol. 2016;36 (4):618-626. doi:10.1002/jat.3253

15. Chen ZF, Zhao F, Wang X, et al. Organs distribution and injury after repeated intratracheal instillations of nano- $\operatorname{In}_{2} \mathrm{O}_{3}$ particles into the lungs of Wistar rats. J Nanosci Nanotechnol. 2020;20(3):1383-1390. doi:10.1166/jnn.2020.17173

16. Tanaka A, Hirata M, Matsumura N, et al. Tissue distribution of indium after repeated intratracheal instillations of indium-tin oxide into the lungs of hamsters. J Occup Health. 2015;57(2):189-192. doi:10.1539/joh.14-0123-BR

17. Cummings KJ, Nakano M, Omae K, et al. Indium lung disease. Chest. 2012;141(6):1512-1521. doi:10.1378/chest.11-1880

18. Cummings KJ, Virji MA, Park JY, et al. Respirable indium exposures, plasma indium, and respiratory health among indium-tin oxide (ITO) workers. Am J Ind Med. 2016;59(7):522-531. doi:10.1002/ajim.22585

19. Hawley B, Gibbsa JL, Cummings K, et al. A field evaluation of a single sampler for respirable and inhalable indium and dust measurements at an indium-tin oxide manufacturing facility. J Occup Environ Hyg. 2019;16(1):66-77. doi:10.1080/15459624.2018.1536826

20. Nagano K, Gotoh K, Kasai T, et al. Two- and 13-week inhalation toxicities of indium-tin oxide and indium oxide in rats. J Occup Health. 2011;53 (2):51-63. doi:10.1539/joh.L10128

21. Noguchi S, Eitoku M, Kiyosawa H, Suganuma N. Fibrotic gene expression coexists with alveolar proteinosis in early indium lung. Inhal Toxicol. 2016;28(9):421-428. doi:10.1080/08958378.2016.1193573

22. Jeong J, Kim J, Seok SH, Cho WS. Indium oxide $\left(\mathrm{In}_{2} \mathrm{O}_{3}\right)$ nanoparticles induce progressive lung injury distinct from lung injuries by copper oxide (CuO) and nickel oxide (NiO) nanoparticles. Arch Toxicol. 2015;90(4):817-828. doi:10.1007/s00204-015-1493-x

23. Naji A, Muzembo BA, Yagyu K, et al. Endocytosis of indium-tin-oxide nanoparticles by macrophages provokes pyroptosis requiring NLRP3-ASCCaspase1 axis that can be prevented by mesenchymal stem cells. Sci Rep. 2016;6(1):1-18. doi:10.1038/srep26162

International Journal of Nanomedicine

Dovepress

\section{Publish your work in this journal}

The International Journal of Nanomedicine is an international, peer-reviewed journal focusing on the application of nanotechnology in diagnostics, therapeutics, and drug delivery systems throughout the biomedical field. This journal is indexed on PubMed Central, MedLine, CAS, SciSearch ${ }^{\mathbb{B}}$, Current Contents ${ }^{\mathbb{B}} /$ Clinical Medicine, Journal Citation Reports/Science Edition, EMBase, Scopus and the Elsevier Bibliographic databases. The manuscript management system is completely online and includes a very quick and fair peer-review system, which is all easy to use. Visit http:// www.dovepress.com/testimonials.php to read real quotes from published authors.

Submit your manuscript here: https://www.dovepress.com/international-journal-of-nanomedicine-journal 